\title{
Dante ghibellino. Note per una discussione
}

\author{
Enrico Fenzi
}

enrico.fenzi@aleph.it

\begin{abstract}
Il saggio propone un'ipotesi di cronologia che riesca a precisare i momenti diversi ma certamente molto vicini della richiesta di perdono ai 'neri' fiorentini che è nella canzone Tre donne, e il forte spirito ghibellino che anima l'elogio di Federico II e Manfredi nel De vulgari eloquentia. Di qui, muove la considerazione del progressivo distacco di Dante dalla prospettiva guelfa del maestro Brunetto Latini, e il finale approdo a posizioni apertamente filo-imperiali che l'Inferno non rinnega e non nasconde, come pure qualcuno ha potuto pensare.
\end{abstract}

Parole chiave: Guelfi; Ghibellini; Tre donne; Federico II; Manfredi; Brunetto Latini; Impero.

\section{Abstract}

The essay proposes a chronlogical hypothesis which will clarify the diverse yet related moments of the request for the Black Guelfs' pardon in the canzone Tre donne, and the marked Ghibelline spirit which gives life to the eulogy of Frederick II and Manfredi in De vulgari eloquentia. Dante's progressive estrangement from the Guelf viewpoint of his mentor Brunetto Latini is also considered as it leads to an openly pro-imperial stance that the Inferno neither denies nor hides, as some have indeed thought.

Keywords: Guelfs; Ghibellines; Tre donne; Frederick II; Manfredi; Brunetto Latini; Empire. 
1. Nel De vulgari eloquentia I XII 3-5, subito dopo aver citato con onore le canzoni Ancor che l'aigua per lo foco lassi e Amor, che lungiamente m'hai menato di Guido delle Colonne come esemplari del volgare illustre di Sicilia, ${ }^{1}$ Dante scarta all'improvviso dalla linea maestra del discorso che riprenderà avanti, $\mathbb{S}$ 6 , con la formula «Sed prestat ad propositum repedare quam frustra loqui», e introduce questa eloquente, e famosa, digressione che è opportuno citare per intero:

Sed hec fama Trinacrie terre, si recte signum ad quod tendit inspiciamus, videtur tantum in obproprium ytalorum principum remansisse, qui non heroico more sed plebeio secuntur superbiam. Siquidem illustres heroes, Fredericus Cesar et benegenitus eius Manfredus, nobilitatem ac rectitudinem sue forme pandentes, donec fortuna permisit, humana secuti sunt, brutalia dedignantes. Propter quod corde nobiles atque gratiarum dotati inherere tantorum principum maiestati conati sunt, ita ut eorum tempore quicquid excellentes animi Latinorum enitebantur primitus in tantorum coronatorum aula prodibat; et quia regale solium erat Sicilia, factum est ut quicquid nostri predecessores vulgariter protulerunt, sicilianum vocetur: quod quidem retinemus et nos nec posteri nostri permutare valebunt. Racha, racha! Quid nunc personat tuba novissimi Frederici, quid tintinnabulum secundi Karoli, quid cornua Iohannis et Azonis marchionum potentum, quid aliorum magnatum tibie, nisi "Venite carnifices, venite altriplices, venite avaritie sectatores»? ${ }^{3}$

Una prima rapida glossa. È impossibile sottovalutare questo omaggio a Federico II e Manfredi che, per essere ben radicato e pienamente coerente con

1. Ora da leggere nell'edizione a cura di Corrado Calenda in Poeti della Scuola Siciliana, Milano: Mondadori, 2008, II, p. 86-108. Sulle probabili ragioni di questa scelta vd. Mario Marti, Con Dante fra i poeti del suo tempo, Lecce, Milella, 1966, p. 31-42. Dante però non nomina l'autore e per la verità non ha ancora parlato di 'volgare illustre', per il quale rimanda avanti: infatti il vulgare sicilianum del quale qui tratta, se assunto "secundum quod ab ore primorum Siculorum emanat, ut in preallegatis cantionibus perpendi potest, nichil differt ab illo quod laudabilissimum est, sicut inferius ostendemus» [così come esce dalle labbra dei Siciliani più eminenti, quale si può osservare nelle canzoni già citate, non è per nulla diverso dal volgare che è degno della massima lode, come più avanti mostrerò].

2. [Ma è meglio tornare all'argomento che sprecare parole].

3. [Ma questa fama della terra di Trinacria, se consideriamo con attenzione a quale conclusione ci porta, sembra sopravvivere solo quale marchio d'infamia per i principi italiani, superbi non al modo degli eroi ma a quello dei plebei. In verità quei grandi e illustri signori, l'imperatore Federico e il suo bennato figlio Manfredi, hanno mostrato tutta la nobiltà e rettitudine del loro animo, e finché la fortuna l'ha permesso si sono comportati da veri uomini, rifiutando con spregio di comportarsi da bestie. Proprio per questo chi aveva nobiltà di cuore e abbondanza di doni divini si è sforzato di tenersi a stretto contatto con la maestà di così grandi signori, sì che a quel tempo tutto quello che i migliori degli Italiani producevano nasceva alla corte di quei grandi re. E poiché la Sicilia era la sede regale, è avvenuto che quello che i nostri predecessori hanno prodotto in volgare si chiamasse 'siciliano': cosa che tutti noi accettiamo e che i posteri non potranno mutare. Racà, racà! Cosa suona, ora, la tromba dell'ultimo Federico? Cosa la campana di guerra del secondo Carlo? Cosa i corni dei potenti marchesi Giovanni e Azzo? Cosa i flauti degli altri magnati? Niente altro che: "Assassini, venite a noi! Bugiardi, venite a noi! Servi dell'avidità, venite a noi!»]. 
tutto il discorso che Dante è andato svolgendo sino a quel punto, non cessa tuttavia di sorprendere per il suo tono vibrante, per la sua portata politica clamorosamente ghibellina e per la sua complessa sostanza ideologica, che rinvia alle teorizzazioni sulla nobiltà svolte dieci anni prima nella canzone Le dolci rime e ora riprese e sviluppate in direzione apertamente aristocratica e 'imperiale' nel libro IV del Convivio. ${ }^{4} \mathrm{E}$ di questa complessità e stratificata ricchezza di significati è in fondo prova anche il fatto, già per altro osservato, che un ritratto siffatto ci porta direttamente alla figura di Ulisse. ${ }^{5}$ Dall'altro lato, i principi e i marchesi del tempo presente, che hanno tradito l'alto modello offerto da Federico II e da Manfredi e che Dante insulta con tanta violenza nelle ultime righe del passo, sono nell'ordine Federico II d'Aragona (12721337), incoronato re di Sicilia nel 1296 e riconfermato nel 1302 con la pace di Caltabellotta; Carlo II d'Angiò (1248-1309), figlio del primo Carlo, incoronato re di Sicilia da papa Niccolò IV nel 1289, donde appunto la guerra con il re aragonese; Giovanni di Monferrato, ultimo e, per Dante, degenere erede della dinastia degli Aleramici, che dopo la morte del padre Guglielmo nelle carceri di Alessandria, riparò ancora fanciullo in Provenza e a Napoli, donde tornò in Piemonte nel 1294 per ricostituire il dominio di famiglia, e morì senza eredi nel gennaio 1305; l'ultimo, Azzo VIII d'Este, stretto alleato dei 'neri' fiorentini, succedette al padre Opizzo II nel 1293 e morì nel $1308 .{ }^{6}$

4. Debbo sùbito dire che la più forte e decisa rivendicazione del valore di questa presa di posizione è stata fatta in pagine che restano fondamentali da John A. ScotT, Dante's Political Purgatory, Philadelphia: University of Pennsylvania Press, 1996, p. 30-35, che per primo ha puntualizzato che «It is surely impossible to doubt Dante's vigorously Ghibelline, anti Guelf attitude when he wrote this passage (c. 1304), with its exaltation of the Staufen rulers of Sicily, whose achievement remained to the eternal shame of the contemporary rulers of Italy» (p. 32). Un'altra preziosa indicazione di Scott è nel suo limpido saggio "Il mito dell'imperatore negli scritti danteschi», in Dante. Mito e poesia. Atti [...] a cura di Michelangelo Picone e Tatiana Crivelli, Firenze: Cesati, 1999, p. 89-105, là dove osserva (p. 90): «L'assenza di qualsiasi accenno alla necessità che l'imperatore risieda a Roma, insieme alle aspre critiche mosse ai Romani nel precedente capitolo [cioè in Dve I 11], a noi sembra indicare un momento 'ghibellino' ancora privo del concetto della romanitas dell'Impero, concetto assolutamente fondamentale che invece affiorerà, anzi sgorgherà dal cuore dell'autore del Convivio, per rimanere sempre al centro del pensiero politico del poeta». Ciò, insieme ad altri elementi, conferma come il libro quarto del Convivio sia posteriore al De vulgari eloquentia e mostra insieme la 'velocità' dell'esperienza dantesca in questi anni cruciali. Ma dello stesso Scott si veda anche Dante magnanimo. Studi sulla "Commedia», Firenze: Olschki, 1977, passim, ma in particolare, per quanto si dirà sulla nobiltà, p. 239-245.

5. Vd. Richard Weigel, «Dante's Ambivalence towards Julius Caesar and Frederick II», Classica et Mediaevalia, n. 51, 2000, p. 271-288: p. 278: «Although the poet was surely familiar with the bizarre tales told about Frederick by Salimbene, Villani and others, he ignores most of these and refers to him with respect instead of caricaturing him as some kind of modern Ulysses, though he does consign the Emperor to Hell». Ma per ciò che comporta l'elogio, vd. ancora avanti.

6. Anche altrove Dante giudica severamente questi che formano una sorta di grottesca orchestrina, e motiva la propria condanna (vd. Giorgio Varanini, s. v. Este, Azzo VIII d', in ED, I, 1970, p. 748): per Federico d'Aragona vd. Conv. IV vi 20; Purg. VII 119-20; Par. XIX 
Molti elementi, tutti diversamente importanti, immediatamente emergono. Il primo: Giovanni di Monferrato, dato come vivente, è morto - s'è appena visto - nel gennaio 1305, e ciò ha permesso da tempo di datare questa parte del De vulgari eloquentia (e forse tutto il primo libro) alla seconda metà del 1304. Fosse o no a Bologna nel biennio 1304-5, come qualcuno ha ipotizzato, ${ }^{7}$ certo è che per Dante si trattò di un momento particolarmente delicato. Proprio all'inizio dell'estate egli si era defintivamete staccato dalla "compagnia malvagia e scempia» dei Bianchi che andavano precipitando verso la disfatta della Lastra (20 luglio), ${ }^{8}$ e ben riusciamo a immaginare quanto una tale rottura fosse traumatica e complessa, anche per le accuse di tradimento che si tirò dietro e i probabili tentativi di ritorsioni e vendette da parte degli ex-compagni, delle quali è ancora l'eco nelle parole dell'Ottimo a proposito dei preliminari della battaglia della Lastra:

et qui tocca come li Bianchi ebboro a sospetto Dante per uno consiglio ch'egli rendee, che l'aiutorio delli amici s'indugiasse di prenderlo nel tempo di verno, alla seguente istate più utile tempo a guerreggiare; il quale consiglio seguitato da' Bianchi non ebbe l'effetto che l'autore credette, però che l'amico poi richesto non prestoe l'aiutorio, onde i Bianchi stimarono che Dante corrotto da' Fiorentini avesse renduto malvagio consiglio. ${ }^{?}$

Molti anni dopo Dante medesimo rievocò tutto questo in Par. XVII 61-69, attraverso le profetiche parole di Cacciaguida che, a dispetto della distanza, restituiscono la drammaticità della situazione allora attraversata, e confermano le vecchie scelte e i durissimi giudizi contro la 'parte' entro la quale aveva militato in posizione di spicco, via via definita malvagia, scempia, ingrata, matta, empia e infine 'bestiale':

E quel che più ti graverà le spalle, sarà la compagnia malvagia e scempia con la quale tu cadrai in questa valle;

130-5 e XX 62-3. Per Carlo II d'Angiò vd. De vulg. el. II vi 4; Conv. IV vi 20; Purg. VII 126-7 e XX 79-81; Par. VI 106-8; XIX 127-9; XX 62-3. Per Giovanni di Monferrato, vd. Purg. VII 133-6. Per Azzo VIII d'Este vd. De vulg. el. II vi 4; Inf. XII 111-2; Purg. V 64-84; XX 80-1.

7. Vd. ora il recentissimo volume di Marco Santagata, Dante. Il romanzo della sua vita, Milano: Mondadori, 2012, in part. p. 389-391. A questo non certo soggiorno bolognese Mirko Tavoni lega in modo forse troppo stretto la composizione del De vulgari eloquentia: vedine l'Introduzione in Dante, Opere, Milano: Mondadori, 2011, I, p. 1113-1116 (osserverei brevissimamente: la Bologna 'bianca' del 1304-5 era il soggiorno adatto per il Dante 'traditore' della sua parte, almeno a far data dalla battaglia della Lastra?). Si troveranno qui e là i necessari complementi bibliografici, tra i quali spicca il fondamentale lavoro di Umberto CARPI, La nobiltà di Dante, Firenze: Polistampa, 2004, passim, che ha cominciato a dare i suoi frutti e del quale anche questo mio intervento si dichiara debitore, come anche degli altri suoi citati più avanti.

8. Robert Davidsohn, Storia di Firenze, Firenze: Sansoni, 1960, IV, p. 394-397.

9. In Giorgio Petrocchi, Vita di Dante, Bari: Laterza, 1986, p. 98 nota 8. 
che tutta ingrata, tutta matta ed empia

si farà contr'a te; ma, poco appresso,

ella, non tu, n'avrà rossa la tempia.

Di sua bestialitate il suo processo

farà la prova, sì ch'a te fia bello

averti fatta parte per te stesso.

Non voglio né posso ricostruire qui un intero capitolo della biografia di Dante sul quale tanto è già stato scritto e che tuttavia resta alquanto oscuro, ma è precisamente nel contesto di un tale distacco dalla propria 'parte' che il passo del De vulgari eloquentia va collocato, aiutandoci a capire quanto fosse profondo il capovolgimento delle posizioni dantesche, tale da spezzare non solo tutta la rete dei rapporti di militanza politica stretti in quei primissimi anni d'esilio, ma anche da ribaltare la passata ideologia comunale e brunettiana della quale s'era nutrito e alla quale aveva sin lì ispirato la sua azione pubblica. Vedremo meglio, nel merito. Intanto, un tale distacco non è la sola cosa che importi sottolineare, perché parrebbe verisimile che allora Dante desse un taglio netto anche alle ambiguità che in forma allusiva e non del tutto decifrabile traspaiono dalla canzone Tre donne, e in particolare dai suoi congedi, e dal secondo in ispecie, forse assente da molti manoscritti non già perché aggiunto più tardi, ma piuttosto perché adombrava un'ipotesi di personale accordo con i Neri troppo acrobatica e allarmante per poter essere partecipata a tutti. ${ }^{10} \mathrm{E}$ in perfetta coerenza con quell'ipotesi di accordo è in ogni caso da leggere nella medesima canzone l'ammissione di una colpa, che non sarà tanto quella di aver rivolto le armi contro la patria (in fondo questo rientrava nei rischiosi diritti di

10. Si tratta di un'ipotesi che già ho formulata nelle ultime righe del saggio: «Tre donne 73-107: la colpa, il pentimento, il perdono", in Juan Varela-Portas de Orduña (ed.), Tre donne intorno al cor mi son venute, Madrid: Departamento de Filología Italiana de la Universidad Complutense de Madrid («Biblioteca de Tenzone»), 2007, p. 91-124: p. 117, e che vedo ripresa e ulteriormente argomentata da CARPI, «Tre donne intorno al cor mi son venute», in c. s. per il secondo volume, imminente, di D. A., Le quindici canzoni. Lette da diversi, Lecce: Pensa Multimedia, che ho potuto leggere per cortesia dell'autore, al quale devo anche la possibilità di leggere, proprio mentre attendo alla stesura di queste mie pagine, l'altro suo saggio, «Da Federico II a Manfredi», in c. s. per gli Atti del Convegno dantesco di Bertinoro, nel settembre 2010 (i due saggi, mi dice l'amico Carpi, usciranno comunque nella primavera del prossimo anno, raccolti con altri nel volume Linferno dei Guelfi e il Purgatorio dei principi, per l'editore Franco Angeli). A questi saggi rimando anche per le necessarie integrazioni bibliografiche, tra le quali spicca, anche per la proposta di individuazione di tre congedi, Guglielmo GorNI, "Filologia e nazionalismo. Tre donne e tre dantisti», in ID., Dante prima della Commedia, Firenze: Cadmo, 2001, p. 222 ss. (ma in contrario, con buone ragioni, Stefano CarraI, «Il doppio congedo di Tre donne intorno al cor mi son venute», in Le Rime di Dante, a cura di Claudia Berra e Paolo Borsa, Milano: Cisalpino ["Quaderni di Acme», 117], 2010, p. 197211). Ma si veda pure, nello stesso volumetto della «Biblioteca de Tenzone», Umberto CARPI, «Il secondo congedo di Tre donne», p. 15-26. Qui Carpi compendia benissimo quanto aveva diposto su una assai più ampia superficie nel volume La nobiltà, cit., e i suoi risultati, con i quali mi dovrò sùbito confrontare, sono poi ripresi e risolti in forma narrativa (e dunque, forzatamente, con qualche ipotesi di troppo) da Santagata, Dante. Il romanzo, cit., specialmente p. 171-172 e p. 183-197. 
un esule), ma piuttosto quella, imperdonabile, di averlo fatto cercando l'accordo con i nemici per antonomasia, i Ghibellini fuorusciti. ${ }^{11}$ Quel congedo, insomma, e l'ammissione della colpa sarebbero di per sé sufficienti a rendere specialmente inquietanti le insinuazioni dell'Ottimo sopra riferite. Ma ecco che il discorso si fa sùbito delicato e richiede un supplemento di riflessione.

2. Lasciamo per un momento da parte il De vulgari eloquentia, che pure resta il perno essenziale del discorso che vorrei fare, e consideriamo invece la ricostruzione di gran lunga più ragionata e attendibile di quegli anni difficili, cioè quella di Carpi. Alla base sta la testimonianza di Leonardo Bruni che nella sua Vita di Dante, composta nel 1436, racconta:

Fallita adunque questa tanta speranza [cioè dopo la battaglia della Lastra], non parendo a Dante più da perdere tempo, partì d'Arezzo et andossene a Verona; dove, ricevuto molto cortesemente da' signori della Scala, co' loro fece dimora alcun tempo et ridussesi tutto a umiltà, cercando con buone opere et con buoni portamenti racquistare la gratia di potere tornare in Firenze per spontanea revocatione di chi reggeva la terra. Et sopra questa parte s'affaticò assai, et scrisse più volte, non solamente a particulari cittadini et del reggimento ma al popolo; et intra l'altre, una epistola assai lunga, che incomincia: «Popule mee, quid feci tibi?». Essendo in questa speranza Dante di ritornare per via di perdono, sopravvenne la electione d'Arrigo di Luzimborgo imperadore, per la cui electione prima, et poi per la passata sua essendo tutta Italia sollevata in speranza di grandissima novità, Dante non potette tenere il proposito suo dell'aspettare la gratia, ma, levatosi co' l'animo altero, cominciò a dire male di quelli che reggevano la terra, appellandoli scellerati e cattivi et minacciando la debita vendetta per la potentia dello imperadore, contra la quale dicea essere manifesto loro non avere alcuno scampo [...] Morto dappoi lo 'mperadore Arrigo, il quale nella seguente state morì a Bonconvento, ogni speranza al tutto fu perduta da Dante, però che di gratia lui medesimo s'avea tolta la via per lo parlare et scrivere contro a cittadini che governavano la repubblica, et forza non ci restava per la quale sperare potesse. Sì che, deposta ogni speranza, povero assai trapassò il resto della sua vita, ecc. ${ }^{12}$

Com’è evidente e come altri passi della Vita confermano, Bruni dispone le cose in modo da alleggerire Firenze dalla responsabilità d'aver mantenuto Dante in esilio (è per esempio significativo che egli non faccia cenno della condanna a morte del marzo 1302), e il peso che toglie alla città lo carica sulle

11. Vd. in particolare CARPI, La nobiltà, p. 509, che aggiorna in maniera assai convincente la vecchia proposta di Cosmo, alla quale s'era fortemente e ripetutamente opposto Barbi: vd. anche l'eccellente ricostruzione della polemica nelle fitte pagine di GorNI, Filologia e nazionalismo, p. 232 ss.

12. In Leonardo BRUni, Opere letterarie e politiche, a cura di Paolo Viti, Torino: UTET, 1996, p. 546-547. Bruni parla di altre lettere da lui viste, probabilmente nella cancelleria della Signoria di Firenze, e specifica più avanti, p. 548, che Dante «fu ancora scrittore perfetto, et era la lettera sua magra et lunga et molto corretta, secondo io ho veduto in alcune epistole di sua propria mano scritte». 
spalle di Dante medesimo. L'elemento centrale del suo racconto sta in quell'immagine di Dante che per anni, dal giorno dopo la sconfitta della Lastra nel 1304 sino all'elezione e alla discesa in Italia di Arrigo VII, eletto imperatore nel 1309 e sceso in Italia per l'incoronazione nell'ottobre dell'anno successivo, avrebbe brigato in ogni modo e a vari livelli per rientrare nella Firenze dei 'neri', e che però, illuso dalla presunta invincibilità di Arrigo, era improvvidamente salito sul carro dell'imperatore condannandosi da sé all'esilio perpetuo, senza aspettare una grazia che Bruni insinua come possibile.

Carpi accetta in pieno questa ricostruzione degli anni 1304-1308, e pur se riesce difficile che Dante conducesse i suoi tentativi stando nella Verona ghibellina degli Scaligeri (meglio certamente, allora, la Treviso di Gherardo e Rizzardo da Camino, «intrinseci» del 'nero' Corso Donati), dall'altra allo studioso riesce di mostrare quali e quante fossero le frequentazioni 'nere' del poeta, a cominciare dai Malaspina e da Guido Salvatico, dei Guidi di Dovadola, senza peraltro che sia possibile documentare un qualche loro rapporto con un eventuale rientro del poeta a Firenze. Tuttavia, di là dalle complicate e spesso inestricabili capriole politiche (che di propriamente politico avevano spesso assai poco) di tante famiglie, specie minori, quelle brighe dantesche avrebbero avuto un concreto punto d'appoggio nella spaccatura emersa all'interno dei 'neri' fiorentini tra gli intransigenti Della Tosa e il più flessibile Corso Donati: e una Donati, Gemma, era la moglie di Dante, che si è supposto fosse tornata in quegli anni a Firenze (è invece improbabile che Dante facesse conto sul troppo breve tentativo di rappacificazione del cardinale Napoleone Orsini, nei primi mesi del 1306, finito del resto nel nulla dopo la sua cacciata da una Bologna ormai filo-'nera' il 23 maggio).

Ora, in questa sede non è indispensabile riferire tutta la fitta rete di osservazioni e ipotesi messe in campo da Carpi per dare sostanza a quanto scrive Leonardo Bruni: meglio è leggere direttamente le sue pagine, straordinarie nel gettare fasci di luce su una realtà difficile e poco conosciuta entro la quale lo studioso è probabilmente l'unico a muoversi con tanta maestria. Il punto essenziale, ben chiaro, resta tuttavia questo: il dipingere gli anni 1304-1308 (esattamente quelli ripetutamente indicati da Carpi nei suoi lavori) come anni condizionati dall'intento di trovare da parte di Dante una via più o meno diretta per rientrare a Firenze, e dunque di trovare un accordo con i guelfi 'neri', comporta il rischio di sottovalutare alcuni momenti importanti del suo percorso e di proporne una visione d'assieme alquanto distorta. Arrivo al punto. Quel passo del De vulgari eloquentia ne risulta per dir così depotenziato e risospinto indietro, e ridotto a un primo 'spunto' legato alla relativamente breve fase caratterizzata dalla alleanza con i ghibellini (il triennio 'bianco'ghibellino, con le parole di Carpi): spunto che "comincerà ad esprimersi compiutamente solo nel corso del Purgatorio». ${ }^{13}$ E simmetricamente, dall'altra

13. CARPI, «Il secondo congedo», cit., p. 20. Su questa linea avanza con decisione ancora maggiore Santagata. 
parte, la canzone Tre donne, che pure è data come perfettamente contemporanea a quell'esaltazione di Federico II e Manfredi, è di fatto spinta in avanti e chiamata a inaugurare la successiva e alquanto ambigua fase 1304-1308 nella quale Dante «ridussesi tutto a umiltà, cercando con buone opere et con buoni portamenti racquistare la gratia di potere tornare in Firenze per spontanea revocatione di chi reggeva la terra». Fermiamoci un attimo, infatti, sui problemi posti dalla data alla quale far risalire un testo così delicato come Tre donne. Tradizionalmente, da Carducci in poi la si è datata al $1302,{ }^{14}$ ma ora Carpi la sposta risolutamente in avanti, fondandosi sui v. 88-90: «Onde, s’io ebbi colpa, / più lune ha volte il sol poi che fu spenta, / se colpa muore perché l'uom si penta»:

Nel suo recente commento Domenico De Robertis ha letto più lune nel senso generico di 'alquanto o parecchio tempo', ma io - Dante vuol dire sempre esattamente quel che dice- preferisco attenermi alla lettera, cioè 'più mesi', 'vari mesi': mesi, ma a far data da qual momento, da quale episodio politico di svolta? Non vedo motivo di dubitare che si tratti del distacco di Dante dalla «compagnia malvagia e scempia» dei fuorusciti ghibellini e 'bianchi' consumatosi subito dopo la battaglia della Lastra (ma divergenze politiche e defatiganti discussioni dovevano durare già da tempo dentro quell'alleanza, come traspare dalla stessa lettera di Dante al cardinale Niccolò da Prato), perciò 'più mesi' dopo il 19/20 luglio 1304, le due giornate della battaglia. Fine del 1304 dunque, ovvero prime settimane del 1305: più lune non può significare altro. ${ }^{15}$

Di primo acchito, non vedo come si possa proporre qualcosa di meglio. Tutto perfetto, dunque, se non fosse che la data proposta coincide esattamente, come già s'è detto, con quella del passo del De vulgari eloquentia citato in apertura. Di questo si dovrà riparlare meglio poco avanti, ma si potrà almeno dire, da sùbito, che non si riesce a pensare nulla di più irriducibilmente ostile verso i guelfi fiorentini, tanto per l'esaltazione intrinsecamente ghibellina di Federico II e Manfredi, quanto per il violentissimo vituperio nei confronti di due diversi ma decisivi pilastri del potere 'nero' quali Carlo d'Angiò ${ }^{16}$ e Azzo d'Este (che aveva sposato, con vergognose modalità di 'acquisto', Beatrice, figlia dell'angioino: vd. Purg. XX 80-81), il quale era legatissimo ai 'neri' Della

14. Così ancora, tra altri, Emilio PAsQuini, Dante e le figure del vero. La fabbrica della Commedia, Milano: Mondadori, 2001, p. 125-127, che però giudica il secondo congedo aggiunto nel 1304, quando Dante abbandona la parte 'bianca': «Proprio in quei giorni del 1304 [quelli della sconfitta della Lastra] Dante aggiunge alla canzone un secondo congedo nel quale, pur senza umiliarsi sino al cedimento [...] adombra l'idea di una quasi impossibile pacificazione». Direi tuttavia anch'io, con altri, che la stretta solidarietà tra l'ammissione della colpa nella quinta stanza e il secondo congedo, che senza quell'ammissione male si capirebbe, parrebbe escludere la possibilità di una aggiunta posteriore.

15. CARPI, «Il secondo congedo», cit., p. 17.

16. Del molto che si potrebbe dire, basti qui riferire il concentrato e famoso giudizio di Giovanni Villani, Nuova cronica, IX 50: «Nel detto anno MCCCII, del mese d'aprile, messer Carlo di Valos fornito in Firenze quello perché era venuto, cioè sotto trattato di pace cacciata la parte bianca, di Firenze si partì». 
Tosa e alleato della famiglia bolognese dei Caccianemici nel rivolgimento antighibellino e anti-'bianco'che portò alla cacciata dei 'bianchi' da Bologna nel febbraio 1306 e che, per la sua parte, tanto contribuì all'irrevocabilità dell'esilio dantesco.

Non è chi non veda, a questo punto, la contraddizione che nasce dal considerare come contemporanei due testi assunti come espressioni di due stagioni di segno opposto: quella 'bianco'-ghibellina dei primi anni d'esilio, cioè il triennio 1302-1304, e quella successiva, 1304-1308, se non dichiaratamente filo-'nera', almeno caratterizzata da un atteggiamento di tipo compromissorio con chi aveva il potere in Firenze. Ma ora Santagata, facendo un passo avanti rispetto alla generale sistemazione di Carpi, la risolve in maniera abile anche se, a mio parere, per nulla convicente. Fatto proprio l'impianto generale messo a punto da Carpi, è precisamente sulla data della canzone Tre donne che lo studioso interviene. Come? Carpi, abbiamo visto, contava le più lune a partire dal voltafaccia di Dante che aveva rifiutato di partecipare alla battaglia della Lastra, assunto come concreta prova del pentimento che avrebbe cancellato la precedente colpa dell'alleanza con i ghibellini. Santagata invece, le conta a partire dalla richiesta di perdono contenuta nella lettera Popule mee ricordata dal Bruni. Non ricevendo risposta, Dante si sarebbe risolto a scrivere la canzone per sollecitarla:

I parecchi mesi vanno contati dal momento in cui lui si era pubblicamente pentito, cioè da quando aveva inviato l'epistola ai priori, e pertanto questa canzone deve risalire almeno agli ultimi mesi del 1306, durante il soggiorno in Lunigiana. I versi finali si presentano come una sorta di sollecito, una perorazione indirizzata a chi poteva sostenere la causa di Dante. ${ }^{17}$

È vero: qui e là Dante chiede perdono, e la circostanza è tale che il rapporto tra i due testi va preso in seria considerazione. Ma la lettera, "assai lunga», è perduta, e per di più non conosciamo in alcun modo né l'occasione (se una precisa occasione ci fu) né la data nella quale sarebbe stata scritta. In teoria potremmo addirittura scendere sino al 1308 ... Ma il fatto è, tornando al punto, che l'ipotesi appare strumentalmente intesa a sanare l'imbarazzante coincidenza temporale di due testi in tutto e per tutto opposti, fatti rappresentativi di due fasi distinte della vicenda dantesca. Delle quali la seconda finirebbe per essere affatto regressiva, un vero e proprio momento di stallo nel percorso di Dante, come Santagata, che torno a citare per la sua estrema chiarezza, arriva a dire:

Durante la composizione del Convivio e del De vulgari eloquentia [...] egli aveva mutato notevolmente le sue idee intorno all'impero - che comincia a considerare necessario affinché gli uomini possano conseguire la felicità terrena- e aveva espresso giudizi lusinghieri proprio su quelli che la Chiesa considerava i suoi peggiori nemici [leggi: Federico II e Manfredi]. Ma di questo 
atteggiamento filo-imperiale l'Inferno scritto dopo il 1306 non serba tracce. Non che Dante abbia cambiato idea e sia regredito alle posizioni ideologiche anteriori all'esilio, ma, in un certo senso, si è censurato. Diciamo che, per ottenere l'amnistia personale, ha cercato di fornire un'immagine di sé politicamente corretta e, nello stesso tempo, ha ritenuto inopportuno manifestare le nuove convinzioni sul rapporto tra impero e papato che era andato maturando. ${ }^{18}$

Ecco, non credo in alcun modo che si possano presentare così le cose, e ho l'impressione che l'ancora inedito saggio di Carpi: "Federico II e Manfredi» (vd. sopra, nota 9) sia dedicato precisamente a correggere una tale deriva. Certo, Carpi accenna alla "convulsa contraddittorietà» delle esperienze politiche di Dante e, in quegli anni, a una «fase complicata di intricatissima geografia e psicologia politica», ma pure puntualizza che «è ben chiaro che la lode a Federico e a Manfredi nel De vulgari è politicamente sostanziale, esprime una precisa, articolata idea geografica e linguistica dell'Italia e della sua collocazione in Europa [...] quella chiara visione del disgregato spazio della 'lingua di sì da unificare particolarmente dentro una ricostituita compagine imperiale, a sua volta da riportare come aula centrale nella sede naturale di Roma, costituisce una novità sconvolgente nel Dante ormai fuori da Firenze e dal suo esclusivo orizzonte comunale» (p. 8). ${ }^{19}$ Si tratta insomma, come Carpi continuamente ribadisce, di un punto di non ritorno che esclude "l'idea di un percorso ideologico zigzagante», e mostra invece una coerenza e una continuità che non ha nulla a che fare con la necessità tutta contingente di muoversi sfruttando gli eventuali spiragli che facessero balenare una possibilità di rientrare in Firenze, e che a noi può presentare qualche puntuale difficoltà per la ricchezza delle sue interne dinamiche, non certo per atteggiamenti compromissori e opportunistici di un Dante che per tutto il corso dell' Inferno avrebbe 'tradito' se stesso. Che sia in gioco proprio l'Inferno è infatti evidente, specie se si sottolinea, come è stato fatto, che sarebbe solo nel Purgatorio, sin dai suoi primi canti, che finalmente si scoprono le carte del Dante ghibellino e filo-imperiale sin lì ancora nascoste negli incompiuti De vulgari eloquentia e Convivio. Il tema, se affrontato in tutta la sua ampiezza, oltrepassa sùbito l'àmbito di queste pagine ma, molto brevemente e ancora con l'aiuto di Carpi, qualcosa vorrei dire, cominciando dalla data di Tre donne.

Ho già detto che la lettura di Carpi mi sembra ineccepibile nel legare la stesura della canzone ai mesi certo terribili della primavera-estate del 1304 (il che significa pure che abbassarne la data in altri momenti e in altri contesti

18. Ibid., p. 218.

19. Debbo dire che ho esitato un poco a citare da un testo ancora inedito, ma mi sono convinto a farlo perché la sua importanza ai fini del discorso che cerco di svolgere è grande, e perché non mi riesce di censurare come non esistesse un testo che non solo ho sott'occhio, ma che è sopraggiunto a condizionare direttamente quanto vado scrivendo. Posso infine aggiungere che il saggio è composto di 21 cartelle piuttosto fitte, e che i numeri di pagina che indico hanno, naturalmente, un mero valore indicativo. 
pare improprio e, in ogni senso, 'fuori tempo'). ${ }^{20} \mathrm{Ma}$ è anche fondamentale chiarire che la canzone è un testo di tale peso da non essere riducibile a un episodio da legare a una occasionale strategia di rientro in Firenze che non contraddice altre più motivate e profonde linee di pensiero, e che la sua stretta contemporaneità con le dichiarazioni del De vulgari eloquentia relative a Federico II e Manfredi, raddoppiate con quelle relative a Carlo d'Angiò ed Azzo d'Este, non la si può digerire a cuor leggero. Allora, che pensare? Se cerchiamo di intendere e rispettare un percorso politico, ideologico e morale di tanta forza come quello dantesco (e non certo per ragioni di lesa maestà, anche se riconosco che, nel caso, potrebbero avere qualche spazio), e dunque escludiamo l'ipotesi di una doppiezza davvero inquietante, resta, credo, una sola via di uscita. Torniamo alle più lune, cioè ai mesi passati dal momento in cui il pentimento ha cancellato la colpa e risentiamo ancora i versi 88-90 della canzone: "Onde, s'io ebbi colpa, / più lune ha volto il sol poi che fu spenta, / se colpa muore perché l'uom si penta», per dire, prima di tutto, che non sappiamo quanti sono i mesi: tre? quattro? cinque? e soprattutto non sappiamo quando è che Dante si sarebbe pentito, e dunque da dove dobbiamo cominciare a contarli. Se la mancata partecipazione alla battaglia della Lastra, in luglio, è stato il frutto di tale pentimento, dobbiamo per forza immaginare una tormentata e convulsa fase precedente durante la quale è maturato e infine esploso il dissenso di Dante nei confronti della propria parte, ${ }^{21}$ e in questo quadro è importante ricordare la testimonianza sopra citata dell'Ottimo, mentre è logico pensare che proprio la linea della trattativa costituisse il nodo concreto del contrasto. A questo punto, ipotesi per ipotesi, ecco quanto azzarderei: Dante, durante questa fase critica, può aver tentato, magari per vie tutte personali, ${ }^{22}$ una trattativa con i 'neri' che costoro hanno respinto e che i suoi,

20. Com'è a mio parere la proposta di abbassare Tre donne alla fine del 1306, e forse oltre, e trovo addirittura curioso che si possa parlare di una rabbiosa reazione degli ex-compagni non già quando Dante si staccò da loro nella primavera-estate del 1304, ma solo due anni dopo, almeno, quando avrebbe mandato la lettera vista dal Bruni (ma così SANTAGata, Dante, cit., p. 172 e 190).

21. Qualcosa al proposito lo si può dedurre dalla lettera scritta a nome di Alessandro conte di Romena, capitano della Fraternità dei 'bianchi' e dei ghibellini, al cardinale 'paciaro' Niccolò da Prato nel marzo-aprile 1304. In apertura Dante prega infatti il cardinale di scusare il ritardo della risposta, considerando «quantis qualibusque consiliis et responsis, observata sinceritate consortii, nostra Fraternitas decenter procedendo indigeat» (Epist. I 2 [di quante e quali discussioni e risposte la nostra Fraternità abbia bisogno per poter procedere correttamente, fatta salva la sincerità del legame che ci unisce]).

22. Si può pensare ai legami di parentela che univano la moglie Gemma a Corso Donati, e si può immaginare altro. Piacerebbe pensare per esempio a Betto Brunelleschi, d'origine ghibellina ma potente capo dei 'neri' ch'ebbe tra l'altro, proprio nel luglio 1304, un ruolo, per quanto del tutto teorico, di paciere: ma ora De Robertis, sulle orme di Gorni, preferisce individuare il Brunetto dell' incipit del sonetto dantesco Messer Brunetto, questa pulzelletta ( ${ }^{\circ}$ 49; Barbi, XCIX) non in Betto Brunelleschi, come si faceva sull'autorità della didascalia del Vat. Lat. 3214, ma in Brunetto Latini. Non si va comunque oltre le mere congetture, per altro autorizzate dalle convulse vicende del 1304. Ma si può almeno ricordare che per opera di Niccolò da Prato e sotto la sua protezione tra il maggio e il principio di giugno 
i 'bianchi', hanno sconfessato, fiutandovi per di più l'odore del tradimento (come sempre accade a chi nel calore della lotta si fa portatore di istanze simili). Egli si è dunque ritrovato perfettamente, irrimediabilmente solo. E proprio questa situazione di isolamento ci autorizza a leggere in chiave 'politica' le parole di Cacciaguida nel XVII del Paradiso, nelle quali Dante, a ritroso, rovescia e sublima in un atto di scelta ciò che fu, invece, una vera e propria espulsione dallo scenario dello scontro. Tutto questo, di là dalla verisimiglianza della cosa, lo si dice anche per guadagnare, in tanta incertezza, un poco di tempo. Le più lune, insomma, potrebbero essere contate non già dal luglio 1304, ma da qualche momento precedente nel quale doveva essere stato chiaro ai 'neri', per contatti diretti o indiretti, che l'atteggiamento di Dante era mutato e che di ciò aveva dato garanzia. Di qui, mesi dopo e consumatosi il distacco, Dante, sospettato di tradimento dai suoi, espulso da ogni schieramento e ormai affatto privo di riconosciuta identità politica, non ha in effetti altra via che quella, dettata dalla passione e dalla disperazione, di volgersi ai 'neri' con Tre donne e di rivendicare dinanzi a loro, le proprie passate posizioni. E lo fa, naturalmente, alla grande, costruendo quello che potremmo anche chiamare l'ossimoro che divide in due la canzone: le stanze 1-4 sono dedicate all'alta sacralità delle tre donne, depositarie dei valori destinati a rifluire su di lui che, fatto forte di tale investitura, nella stanza 5 e nei due congedi riesce ad affrontare in nome della giustizia e della virtù offese i nodi della propria situazione, e a rimettere in mano ai 'neri' la possibilità della loro soluzione.

3. Se l'ipotesi appena sopra abbozzata tiene, e ci riesce dunque di far stare Tre donne entro, che so? il settembre, o anche prima (con quel più lune potremmo addirittura pensare che Dante abbia interesse a enfatizzare un periodo non più lungo di due mesi), saremmo riusciti a fare un poco di spazio, per quanto sempre assai compresso, attorno a quel passo del De vulgari eloquentia, al quale è finalmente il momento di tornare. E che di un poco di spazio ci sia bisogno è dimostrato dalla circostanza che il discorso su Federico II e Manfredi non erompe dal nulla, ma è il risultato di un pensiero che sin dalle prime righe del trattato è cresciuto su se stesso, si è dilatato (per usare un verbo caro a Dante), e una volta imboccata la giusta direzione ha bruciato le tappe, facendo saltare molti ponti dietro di sé. Con tutto lo strazio e le inevitabili aderenze sentimentali imposte dalle circostanze, infatti, è chiaro che il Dante del De vulgari eloquentia, e dunque il Dante dell'autunno-inverno del 1304, si è fatto una ragione dell'esilio ed ha ormai deciso intorno alla propria condizione di 'cittadino del mondo'. Ciò comporta che sparisca il pur cauto accenno che è ancora in Convivio I III (per non dire del momento di forte sbandamento che

erano rientrati in Firenze per trattare la pace un buon numero di fuorusciti ghibellini già condannati a morte, e che è probabile che, se davvero Dante si è compromesso in qualche tipo di trattativa con i 'neri', l'abbia fatto appunto nell'àmbito dei tentativi messi inutilmente in opera dal cardinale (vd. Davidsohn, Storia, IV p. 383-386 per il soggiorno fiorentino dei capi ghibellini, e p. 393 per la missione di pace di Betto Brunelleschi). 
ha prodotto Tre donne), ove si ha l'impressione che insieme alle commosse note di carattere personale: la povertà, per esempio, e l'invilimento ..., s'accompagni ancora una sia pur vaga speranza di ritorno a Firenze nel cui «dolce seno» egli desidera "con tutto lo core di riposare l'animo stancato e terminare lo tempo ch'è m'è dato». Diversamente, direi, nel famoso capitolo sesto del primo libro del De vulgari eloquentia l'esilio è ormai contemplato come destino, e addirittura si rovescia precisamente nella condizione dolorosa ma privilegiata che di là dai miseri confini municipali permette di contemplare dall'alto, sub specie linguistica, popoli e nazioni:

Nos autem, cui mundus est patria velut piscibus equor, quanquam Sarnum biberimus ante dentes et Florentiam adeo diligamus ut, quia dileximus, exilium patiamur iniuste, rationi magis quam sensui spatulas nostri iudicii podiamus. Et quamvis ad voluptatem nostram sive nostre sensualitatis quietem in terris amenior locus qual Florentia non existat, revolventes et poetarum et aliorum scriptorum volumina quibus mundus universaliter et membratim describitur, ratiocinantesque in nobis situationes varias mundi locorum et eorum habitudinem ad utrunque polum et circulum equatorem, multas esse perpendimus firmiterque censemus et magis nobiles et magis delitiosas et regiones et urbes quam Tusciam et Florentiam, unde sumus oriundus et civis, et plerasque nationes et gentes delectabiliori atque utiliori sermone uti quam Latinos. ${ }^{23}$

In qualche maniera si può allora pensare che lo stacco che divide le affermazioni del primo libro del Convivio da quelle appena viste del De vulgari eloquentia (che, ricordiamo, è stato scritto dopo il terzo del Convivio) ${ }^{24}$ riproduca almeno in parte lo stesso stacco che divide Tre donne dall'altro passo del De vulgari eloquentia, quello appunto relativo a Federico II e Manfredi. Ed è certo per altro che il giudizio sul siciliano illustre e su Federico II e Manfredi e, di più, il nesso di consustanzialità che Dante coglie tra quel volgare e quella curia nella quale si scopre che il volgare illustre, una volta definito come tale, aveva trovato il proprio adeguato ubi consistam politico e sociale (si vada a Dve I xviII), è frutto dell'allargamento e, propriamente, dello sfondamento prospettico testimoniato con tanta forza dal precedente capitolo sesto. Aggiungendo che la natura politica

23. [Dve I vi 3: Ma io, che ho per patria il mondo come i pesci hanno il mare, benché abbia bevuto nell'Arno prima di mettere $i$ denti e ami Firenze a tal punto da patire ingiustamente l'esilio proprio per averla amata, regolerò la bilancia del giudizio più sulla ragione che sul sentimento. Certo, per la mia felicità e per la soddisfazione delle mie esigenze personali non esiste in terra luogo più bello di Firenze, ma sfogliando più e più volte i volumi dei poeti e degli altri scrittori che descrivono il mondo sia nell'insieme sia nelle sue singole parti, e analizzando dentro di me le varie localizzazioni delle regioni del mondo e la loro posizione rispetto ai due poli e al circolo equatoriale, ho verificato e posso ribadire che ci sono molte regioni e città più nobili e più belle della Toscana e di Firenze, di cui sono nativo e cittadino, e che ci sono vari popoli e genti che utilizzano una lingua più piacevole e più utile di quella degli italiani].

24. Vd. in particolare Enrico FenZI, «Introduzione», p. XLVII-L, in D. A., De vulgari eloquentia, a cura di E. F., con la collaborazione di Luciano Formisano e Francesco Montuori, Roma: Salerno Editrice («Opere di Dante», III), 2012. 
del De vulgari eloquentia è contenuta per intero in questo gesto di inaudita novità e coraggio che fa dell'esilio un acquisto per sempre. Al proposito, si è giustamente detto e scritto di una concezione dantesca che si sta aprendo all'idea dell'impero, superando gli stretti confini, seppur a suo tempo nutritivi, del repubblicanesimo brunettiano. E infatti, senza rievocare come pure si dovrebbe la demonizzazione di Federico II ad opera della Chiesa e in particolare dei Francescani, che ne avevano fatto il novello Satana e l'incarnazione dell'Anticristo e, nelle figurazioni di scuola gioachimita, la settima testa del drago apocalittico, ${ }^{25}$ si torni proprio a Brunetto, o meglio a uno pseudoBrunetto altrettanto guelfo - il che nel caso nostro non sposta troppo le cose- ${ }^{26}$ essenziale per collocare l'elogio di Dante contro il vero e perdurante sfondo della dura condanna di Federico e di suo figlio quali nemici mortali della Chiesa. E proprio il Tresor, in specie, raccoglie e accredita le più infamanti accuse a carico di Manfredi, che avrebbe assassinato il padre ammalato (I 97, 6: «il entra .1. jour en la chambre ou ses peres gisoit malades et prist.1. grant coussin et le mist sor la face de son pere, et il se coucha sor le coussin, et le fist morir en tel maniere») e avvelenato il fratello Corrado, ${ }^{27}$ sì che a Benevento, ove fu sconfitto e ucciso, a vincere sarebbero stati «li champion Jhesucrist», nella persona di Carlo I d'Angiò e del suo esercito (I 98, 7). Certo, come a proposito di Federico non si può non ricordare la sua lapidaria condanna tra gli eretici nel decimo dell'Inferno, così non si può non ricordare come Manfredi nel terzo del Purgatorio ammetta i suoi orribili peccati. Ma non è qui il caso di allargare il discorso alla Commedia (lo farò, ma in chiave diversa e limitata poco avanti) che in parte sopravviene a riequilibrare il giudizio del De vulgari eloquentia nel suo estremismo del tutto opposto a quello di Tre donne (e diverso, ché il primo è un estremismo ideale, di pensiero,

25. Vd. le dense pagine di Hubert Houben, Federico II. Imperatore, uomo, mito, Bologna: il Mulino, 2009, p. 139-153 (ov'è sùbito da leggere l'inizio dell'enciclica di Gregorio IX del giugno 1239, poco dopo la seconda scomunica dell'imperatore), e le tavole fuori testo 19-21. Vd. anche Wolfgang Stürner, Federico II e l'apogeo dell'impero, Roma: Salerno Editrice, 2009, p. 919-920; Fulvio Delle Donne, Federico II: la condanna della memoria. Metamorfosi di un mito, Roma: Viella, 2012, p. 28 ss. e 43-44. Gioacchino da Fiore, morto nel 1202, ebbe buoni rapporti con Enrico VI e soprattutto con «Costanza imperadrice» (Purg. III 113), madre di Federico VII.

26. Il ritratto e le vicende relative a Federico II e Manfredi, sino a Tagliacozzo e alla morte di Corradino, sono infatti in Tresor I 94-8, capitoli che la recente edizione a cura di Pietro Beltrami et alii, Torino: Einaudi, 2007, ha espunto come aggiunti in un secondo tempo, e dunque ancora da citare nell'edizione Carmody, Berkeley and Los Angeles, University of California Press, 1948, p. 75-81. Resta intatta in ogni caso l'intera, totale fede guelfa e angioina di Brunetto, in rapporto diretto con Carlo di Valois e protonotario del vicario reale Jean Britaud, al quale attribuisce gran parte del merito per la vittoria di Tagliacozzo. Sul punto, e in generale, vd. Enrico FenzI, «Brunetto Latini, ovvero il fondamento politico dell'arte della parola e il potere dell'intellettuale», in A scuola con ser Brunetto. Indagini sulla ricezione di Brunetto Latini dal Medioevo al Rinascimento. Atti [...], a cura di Irene Maffia Scariati, Firenze: Edizioni del Galluzzo, 2008, p. 323-369.

27. Vd. anche il guelfo Giovanni Villani, Nuova cronica, VII 44 e 45, ove si racconta del tentativo di Manfredi di far avvelenare anche Corradino, giovanissimo figlio di Corrado. 
e l'altro, quello di Tre donne, come dire? un estremismo di situazione). Restiamo dunque al momento nel quale il percorso di Dante ebbe la sua oscillazione massima, che ha finito per immetterlo per sempre nel grande solco dell'impero.

Il passaggio dallo scrutinio linguistico all'esaltazione degli imperatori Svevi è netto e conseguente. La rinomanza dei poeti siciliani, dice Dante, non vale per sé, ma va riportata alla fama della Sicilia tutt'intera e, concretamente, alla fama di un suo memorabile e concluso momento di splendore politico e culturale che ha affascinato e al quale hanno collaborato tutti gli Italiani migliori. Il richiamo a tale grandezza, nel presente, serve soprattutto quale termine di confronto che smaschera la miserabile volgarità dei prìncipi italiani, tra $\mathrm{i}$ quali vanno compresi gli stranieri che regnano in Italia, cioè, come dice Conv. IV vi 20: «voi che le verghe de' reggimenti d'Italia prese avete». Tale mossa è in effetti eclatante, quale prima e compiuta testimonianza di un Dante ghibellino che con premeditata violenza sovverte la linea portante del guelfismo fiorentino e osa l'elogio più alto, attribuendo agli «illustres heroes Fredericus Cesar et benegenitus eius Manfredus» quell'eroismo' che i principi italiani non hanno. Per il concetto basta, infatti, volgersi a Tommaso, In Eth., vil lect. 1 1298, commentando Aristotele, Eth. Nic., vil 1 1145a 18-22:

Dicit ergo [Aristotele] primo, quod bestialitati convenienter dicitur opponi virtus, quae communem modum hominum excedit et vocari potest heroica seu divina. Heroas enim gentiles vocabant animas defunctorum virorum insignium, quos etiam esse deificatos dicebant. ${ }^{28}$

Ma non solo 'eroi', e dunque proiettati in una dimensione più che umana, "heroica seu divina», dato "ch'elli son quasi dei», per dirla con Le dolci rime

28. [Dice dunque che alla bestialità efficacemente soppone quella virtù che oltrepassa la comune misura umana e può essere detta eroica o divina. E infatti i gentili definivano come eroiche le anime dei morti più insigni, che dicevano addirittura essere stati assunti tra le divinità]. Su questa linea che associa l'eroe' al semidio vd. anche Uguccione, Derivationes, a cura di Enzo Cecchini et alii, Firenze: Edizioni del Galluzzo, 2004, p. 559: H 16, s.v. her, per il quale l'eroe "pro fortitudine et sapientia sua celo dignus est» [per la sua fortitudine e sapienza è degno del cielo], mentre Dante ha riecheggiato le parole di Tommaso in Conv. III VII 7, e qui ancora, in IV v 12 e 17, esalta coloro che fecero grande Roma come uomini divini. Aggiunge giustamente ad 'eroi' la connotazione di 'fondatori' Roberto ANTONELLI, "La scuola poetica alla scuola di Federico II», in Federico II e le scienze, a cura di Pierre Toubert e Agostino Paravicini Bagliani, Palermo: Sellerio, 1994, p. 309-323: p. 313 (corsivi dell'autore): "senza il grande committente e organizzatore Federico, senza la sua politica culturale, la poesia della scuola siciliana e di Giacomo da Lentini non sarebbe mai esistita, poiché non si sarebbero date le condizioni politiche e storico-culturali per la sua esistenza. Che Federico sia stato mediocre poeta non significa dunque nulla ai fini di questo particolare problema [...]. Al suo nome, in quanto simbolo del poetare, va ascritta l'origine prima della poesia italiana [...] quando in De vulgari eloquentia i, xii, in un capitolo splendido e famoso, Dante spiega perché 'tutto quanto gli Italiani producono in fatto di poesia si chiama siciliano', Federico e Manfredi sono chiamati per nome, io credo, proprio in quanto illustres heroes, 'fondatori'". 
114: essi sono anche 'illustri', sì che della natura divina essi hanno anche la capacità di 'illuminare' le realtà inferiori, secondo l'accezione che più avanti, in Dve I XVII 2 sarà applicata al volgare:

Per hoc quoque quod illustre dicimus, intelligimus quid illuminans et illuminatum prefulgens: et hoc modo viros appellamus illustres, vel quia potestate illuminati alios et iustitia et karitate illuminant, vel quia excellenter magistrati excellenter magistrent, ut Seneca et Numa Pompilius. ${ }^{29}$

Infine, con le parole di Dante, tali principi «humana secuti sunt, brutalia dedignantes», finendo così per stringere in un sol nodo 'umanità' e 'divinità', e riportandoci a Tommaso che nel paragrafo che immediatamente segue le parole sopra citate continua affermando che l'uomo può corrompersi e precipitare al livello delle bestie, oppure esaltare le proprie capacità razionali sì da raggiungere un grado di perfezione «quasi ad similitudinem substantiarum separatum; et haec nominatur virtus divina supra humanam virtutem et communem» (ma naturalmente è inevitabile citare almeno Inf. XXVI 118-120: "Considerate la vostra semenza: / fatti non foste a viver come bruti, / ma per seguir virtute e canoscenza»). ${ }^{30}$ Federico II e Manfredi costituiscono dunque un modello ultimo di perfezione e nobiltà, tale che nelle parole con le quali Dante lo definisce si attua una sorta di importante inversione concettuale: quasi si dicesse non tanto che l'imperatore ha il dovere d'essere compiutamente 'nobile', come tutta l'ampia precettistica al riguardo insegnava, ma piuttosto che chi è dotato di una nobiltà così sublime è di per sé un imperatore. Il che è del resto perfettamente coerente con la concezione sviluppata nel quarto del Convivio a partire dalla canzone di dieci anni prima, secondo la quale la nobiltà è una grazia che solo Dio concede «a l'anima di quelli cui vede stare perfettamente ne la sua persona, acconcio e disposto a questo divino atto ricevere» (Conv. IV xx 7), dal momento che proprio e solo l'infusione di tale grazia definisce un atto di 'elezione' divina che legittima e addirittura invoca ulteriori umane elezioni. ${ }^{31}$

29. [Quando usiamo il termine 'illustre' vogliamo significare qualcosa che illumina e che, colpito dalla luce, risplende. Allo stesso modo chiamiamo 'illustri' certi uomini o perché, illuminati dal potere, riversano su altri luce di giustizia e carità, oppure perché, educati in modo eccellente, sanno educare altrettanto bene, come Seneca e Numa Pompilio]. L'accostamento è già in ScotT, Dante's Political Purgatory, cit., p. 31.

30. [quasi a somiglianza delle sostanze angeliche, e questa è chiamata virtù divina, superiore a quella umana e comune]. Per il richiamo a Ulisse, vd. sopra, nota 5, la citazione di Weigel. Per l'orizzonte concettuale di queste affermazioni dantesche, vd. il grande libro di RenéAntoine Gauthier, Magnanimité. Lidéal de la grandeur dans la philosophie païenne et dans la théologie chrétienne, Paris: Vrin, 1951, partic. p. 295 sgg. per la posizione di Tommaso. Con riguardo a Dante, vd. Fiorenzo Forti, Magnanimitade. Studi su un tema dantesco, Roma: Carocci, 2006 (1977), partic. il cap. I, Il Limbo e i Megalopsicoi della 'Nicomachea' (ma il passo del De vulgari eloquentia non è qui considerato).

31. Andrea Robiglio, "The Tinker as a Noble Man (bene natus) and Preliminary Remarks on the Medieval Concept of Nobility», Vivarium, n. 44, 2006, p. 205-247: p. 242-243: osserva come in Dante "such a notion admits, to some degree, an ontological hierarchy of 
Molto altro si potrebbe dire, specie allargando l'arco dei riferimenti, ma bastino ancora poche cose. Per esempio, va sottolineato con forza il coté volutamente e persino provocatoriamente polemico di un elogio che sovvertiva la maligna vulgata guelfa sulla nascita illegittima di Manfredi, da Dante definito benegenitus quando Brunetto (proprio lui, questa volta), come molti altri, scriveva in Tresor I 93, 2 di «Manfroi, li fiz dou devant dit Frederic, non mie de droit mariage». ${ }^{32} \mathrm{E}$ va ugualmente dato rilievo al fatto che Dante nella canzone Doglia mi reca, la cui composizione cade vicinissima a quella del De vulgari eloquentia, ov'è citata in II II 8 quale testo che merita al suo autore il titolo di 'poeta della rettitudine', si sia probabilmente rifatto per la lunga metafora dei v. 106-116 relativa l'addestramento del falcone, a quell'opera straordinaria che è il De arte venandi cum avibus di Federico II, con l'aiuto del quale riusciamo in effetti a capire meglio le difficili allusioni dantesche. ${ }^{33}$

Dante, insomma, coltiva l'immagine di una curia che era stata capace di calamitare i migliori ingegni d'Italia e di raccoglierli entro una prospettiva politica e culturale unificante, sì che gli excellentes Latinorum avevano trovato alla corte sveva l'occasione unica e irripetibile per esaltare le loro qualità. E avrà dunque anche pensato alla grande politica di incremento degli studi

the human being grounded in the use of 'philosophical' reason, connecting nobility with intellectual virtue. Moreover, the conceptualisation of emotions and human sensualitas plays a vital role: one of the conditions for becoming a noble thinker, in fact, is to be wellborn; that is to say, to have a better mind-body configuration which facilitates the control of passions».

32. Sul significato dell'espressione bene genitus/natus vd. Andrea A. Robiglio, «Dante "bene nato". Guido Cavalcanti e Margherita Porete in Par. V, 115», L'Alighieri, n. 26, 2005, p. 45-62: in part. p. 50-55. Per parlare di Manfredi occorrerebbe andare al canto III del Purgatorio, e alle varie e complesse implicazioni dei versi di Dante. Qui, torno a rinviare alle pagine di Scotт, Dante's Political Purgatory, cit., p. 84-90, ove opportunamente lo studioso insiste su Manfredi quale autore della traduzione dall'ebraico di una operetta in lode delle virtù e della filosofia che Dante potrebbe aver conosciuto, il Liber de pomo sive de morte Aristotilis, pubblicato in edizione critica a cura di Paolo Mazzantini in appendice alla bella lettura di Bruno NARDI, Il canto di Manfredi, del 1960, alla quale rimando: vd. ora i due testi in B. N., "Lecturae» e altri studi danteschi, a cura di Rudy ABARDO, con saggi introduttivi di Francesco Mazzoni e Aldo Vallone, Firenze: Le Lettere, 1990, p. 91-103 (la lettura), e p. 104-125 (Liber de pomo). Nel Prologus Manfredi torna ripetutamente sul motivo che l'uomo, quanto più conosce, tanto più s'avvicina a Dio, e che quanto più è preda della concupiscenza e dei vizi, tanto più assomiglia alle bestie. Per Manfredi e Dante si veda anche Arsenio Frugoni, Scritti su Manfredi, con una presentazione di Enrico Pispisa, Roma: Istituto Storico Italiano per il Medio Evo, 2006, passim.

33. Per ciò rinvio a Enrico Fenzi, «Tra etica del dono e accumulazione. Note di lettura alla canzone dantesca Doglia mi reca», in Umberto CARPI (ed.), Doglia mi reca ne lo core ardire, a cura del Grupo Tenzone, Madrid: Departamento de Filología Italiana UCM/Asociación Complutense de Dantología, 2008, p. 147-211: in part. p. 159-162, con vari riscontri con l'opera di Federico II da leggere in Federico II DI Svevia, De arte venandi cum avibus. L'arte di cacciare con gli uccelli, edizione e traduzione italiana del ms. lat. 717 della Biblioteca Universitaria di Bologna collazionato con il ms. Pal. Lat. 1071 della Biblioteca Apostolica Vaticana, a cura di Anna Laura Trombetti Budriesi, prefazione di Ortensio Zecchino, Roma-Bari: Laterza, 2000. 
messa in opera da Federico II, come tra gli altri scrive lo pseudo-Jamsilla, secondo il quale l'imperatore

philosophiae studiosus erat et quam et ipse in se coluit et in Regno suo propagari ordinavit. Tunc quidem ipsius felici tempore in Regno Siciliae erant literati pauci vel nulli; ipse vero imperator liberalium artium et omnis approbatae scientiae scholas in Regno ipso constituit, doctoribus ex diversis mundi partibus per praemiorum liberalitatem accitis constitutoque tam eis salario quam puperibus auditoribus, sumputum de sui aerarii largitate, ut omnis conditionis et fortunae homines nullius occasione indigentiae a philosophiae studio retraherentur. ${ }^{34}$

Per quanto rapido, il percorso che porta alla 'svolta ghibellina' di Dante è dunque chiaro e irreversibile, e nel corso del De vulgari eloquentia supera altre verifiche e trova altre conferme. L'intero scrutinio delle varianti regionali del volgare è ormai ancorato allo schema federiciano, e il volgare medesimo del quale si va in cerca ne porta in sé il marchio indelebile, come la parte finale del paragrafo solennemente dichiara:

et quia regale solium erat Sicilia, factum est ut quicquid nostri predecessores vulgariter protulerunt, sicilianum vocetur: quod quidem retinemus et nos, nec posteri nostri permutare valebunt. ${ }^{35}$

Non si tratta di un omaggio a una scuola poetica —non solo questoma piuttosto dell'individuazione di un momento fondativo che continuerà ad agire in profondità per tutta l'opera e presiederà alla definizione stessa di 'vol-

34. Nicolaus DE JAMSILla, «Historia de rebus gestis Friderici II imperatoris eiusque filiorum», in Cronisti e scrittori sincroni napoletani editi ed inediti, a cura di Giuseppe DEL RE, Napoli: Stamperia dell'Iride, 1845-1868 (rist. anastatica, Sala Bolognese: Forni, 1976), II, p. 106 [era studioso di filosofia che coltivò in prima persona e che ordinò fosse insegnata nel Regno. Nel suo felice tempo pochi o addirittura nessuno erano i letterati nel regno di Sicilia: fu l'imperatore a fondarvi scuole d'arti liberali e d'ogni scienza riconosciuta facendo venire insegnanti dalle diverse parti del mondo con la promessa di alti compensi e dopo aver stabilito un salario tanto per loro quanto per gli studenti bisognosi, pagato a carico dell'erario, affinché uomini d'ogni condizione e fortuna non fossero distolti dallo studio della filosofia dalla loro povertà]. In questo campo l'iniziativa più clamorosa fu la fondazione nel 1224 dell'Università di Napoli che Federico volle sùbito mettere in concorrenza con Bologna cercando addirittura di strappare a quest'ultima i suoi migliori docenti, specie di diritto: vd. STürner, Federico II, p. 407-418, e in particolare Fulvio Delle Donne, "Per scientiarum haustum et seminarium doctrinarum": edizione e studio dei documenti relativi allo Studium di Napoli in età sveva", Bullettino dell'Istituto storico italiano per il medio evo, n. 111, 2009, p. 101 225. Esalta queste capacità attrattive della corte di Federico II anche il Novellino, XXI: «Lo 'mperadore Federigo fue nobilissimo signore, e la gente ch'avea bontade venìa a lui di tutte parti, però che l'uomo donava volentieri e mostrava belli sembianti a chi avesse alcuna speziale bontà. A lui venieno sonatori, trovatori e belli favellatori, uomini d'arti, giostratori, schermitori, d'ogni maniera gente» (ed. a cura di Cesare SEGRE, in La prosa del Duecento, Milano-Napoli: Ricciardi, 1959, p. 817).

35. [E poiché la Sicilia era la sede regale, è avvenuto che quello che i nostri predecessori hanno prodotto in volgare si chiamasse 'siciliano': cosa che tutti noi accettiamo e che i posteri non potranno mutare]. 
gare illustre'. Il quale volgare è l'unum linguistico-culturale (si veda il decisivo capitolo 16 del primo libro) che adeguatamente corrisponde all'unum politico, e che da questa stessa connessione ricava la connotazione d'eccellenza che ne fa, né più né meno, la lingua elitaria del potere. In altri termini Dante mette qui a fuoco una concezione della lingua che non si potrebbe meglio definire che 'ghibellina', perché fondata su una idealità di tipo ghibellino a tal punto dura verso l'empiria del municipalismo guelfo da mostrare che è stata l'evidenza della diagnosi linguistica a spalancare le porte alla diagnosi politica, che Dante da questo momento in poi non farà che approfondire. Di lì nasce lo schema più volte ripetuto che vede l'effettuale divaricazione tra il linguaggio 'illustre' dei poeti e il linguaggio parlato nelle varie regioni; di lì deriva la forza indirettamente ma intrinsecamente politica con la quale diventa possibile respingere la dialettalità fiorentina; di lì, a chiudere il cerchio, muove la finale definizione del volgare illustre come 'aulico' e 'curiale', cioè consustanziale a una curia, sia essa realmente esistente, come in Germania (Dve I xviII 5: «ut curia regis Alamannie»), oppure ideale ma storicamente attestata come in Italia. Senza dire, poi, che tutto il discorso sui magnalia, nel secondo libro, difficilmente lo si potrà scompagnare da quella fondazione d'eccellenza — ripeto- che proprio all'inizio dell'esame delle parlate italiane impone una volta per tutte le proprie misure.

4. Detto questo, s'accampa di nuovo una questione che oltrepassa il De vulgari eloquentia. Ripetendo, con le parole di Carpi, che la lode a Federico II e a Manfredi è "politicamente sostanziale», ed esprime «una precisa, articolata idea geografica e linguistica dell'Italia e della sua collocazione in Europa», e che da quel punto in poi Dante mai e poi mai avrebbe potuto rinnegare «i punti ormai fermi del proprio sviluppo di pensiero", ${ }^{36}$ occorre anche tornare sul discorso che vedrebbe un guelfismo 'di ritorno' nell' Inferno, e finirebbe per ridurre le affermazioni del De vulgari eloquentia e del libro quarto del Convivio a sortite episodiche e irrelate, in attesa del ritorno esplicito all'ideologia imperiale nel Purgatorio. È evidente che questa non è la sede ove poter affrontare in maniera esaustiva un problema così ampio e intricato, che comporta scontri di opinioni e metodi e sensibilità diverse e dove, in aggiunta, qualsiasi posizione riesce a tirare a sé qualche buona pezza d'appoggio. Mi limiterò dunque a mettere in fila poche osservazioni per modificare almeno in parte quel giudizio che ha trovato l'espressione più estrema nelle chiare parole di Santagata sopra citate, e soprattutto per immergere l'esaltazione di Federico II e di Manfredi in un contesto più ampio, sia all'indietro che in avanti.

La prima dichiarazione in senso lato politica di Dante è nella canzone Le dolci rime dedicata, intorno al 1295,37 a definire cosa sia la nobiltà, con

36. Cito ancora dall'inedito saggio «Da Federico II a Manfredi», p. 8 e 12 del dattiloscritto (vd. sopra, nota 10).

37. Ricordo per scrupolo che sulla base di improbabili argomenti di tipo sentimental-biografico Santangelo giudicava la canzone composta nel 1304 a Bologna, così come Doglia mi reca, 
l'intento insieme polemico e dottrinale di escludere che essa possa dipendere dalle ricchezze, e di trasferire sulle personali qualità dell'individuo quanto era tradizionalmente attribuito alla schiatta. Il discorso di Dante è assai articolato e complesso, ${ }^{38}$ ma a noi qui interessa che sia condotto nei modi della confutazione di una sentenza attribuita a Federico II: «Tale imperò che gentilezza volse, / secondo il suo parere, / che fosse antica possession d'avere / con reggimenti belli» (v. 23-24), nel corso della canzone ulteriormente criticato: «Similemente fu chi tenne impero / in diffinire errato, / ché prima puose 'l falso e, d'altro lato, / con difetto procede» (v. 45-48). ${ }^{39}$ Lasciamo che dieci anni dopo, nel Convivio Dante si preoccupi di mostrare che la sua puntuale critica non intendeva affatto mettere in discussione la «imperiale maiestade e autoritade» (Conv. IV IV 7), che ora difende con passione protestando piena reverenza e soggezione verso tutto ciò che cade sotto la sua giurisdizione, in

Tre donne e Poscia ch'Amor (Salvatore SANTANgelo, Dante e i trovatori provenzali, Catania: Giannotta, 1921, p. 144-156).

38. Su un tema così importante esiste una vastissima bibliografia, entro la quale, in relazione a Dante, va almeno ricordato l'ampio studio di Maria CoRTI, "Le fonti del "Fiore di virtù" e la teoria della nobiltà nel Duecento", Giornale storico della lett. italiana, CXXXVI, 1959, p. 1-82, e il più volte citato volume di CARPI, La nobiltà, specialmente per il primo lungo capitolo, «Il fiorino e la nobiltà», p. 13-321. Ma è ora fondamentale Paolo BorSA, "Sub nomine nobilitatis": Dante e Bartolo da Sassoferrato", in Studi dedicati a Gennaro Barbarisi, a cura di Claudoa Berra e Michele Mari, Milano: CUEM, 2007, p. 59-121, che analizza la celebre repetitio di Bartolo sulla nobiltà, nella quale il grande giurista commenta proprio la canzone dantesca. Sul tema vd. anche i saggi, indispensabili anche per la ricca bibliografia, Andrea Robiglio: "Dante "bene nato" , cit.; "The Tinker as a Noble Man", cit.; "Nobiltà e riconoscimento in Dante: in margine ad una recente edizione del libro IV del Convivio", L'Alighieri, n. 30, 2007, p. 83-102 (si tratta dell'edizione tedesca, Das Gastmahl, diretta da Ruedi Імвасн, Hamburg: Meiner, 2004).

39. Dante non fa però alcun nome, e che si tratti di Federico II lo dice solo anni dopo, nel commento alla canzone in Convivio IV III 6: «Dico dunque: 'Tale imperò', cioè tale usò l'officio imperiale: dove è da sapere che Federigo di Soave, ultimo imperadore delli Romani - ultimo dico per rispetto al tempo presente, non ostante che Ridolfo e Andolfo e Alberto poi eletti siano, appresso la sua morte e delli suoi discendenti-, domandato che fosse gentilezza, rispuose ch'era antica ricchezza e belli costumi». Sin qui non si è trovato da quale fonte Dante abbia potuto attribuire questa definizione a Federico, mentre, come scrive Pernicone, «si può ammettere senz'altro che nell'ambiente dei nobili ghibellini corresse la definizione della nobiltà come antica ricchezza e bei costumi» (in DANTE, Rime della maturità e dell'esilio, a cura di Michele Barbi e Vincenzo Pernicone, Firenze: Felice Le Monnier, 1969, p. 417). In ogni caso è più che normale che l'imperatore l'avesse fatta sua, visto che tale definizione è letteralmente ricavata da Aristotele, Polit. IV 8, 1294 21a: 'la nobiltà è ricchezza antica e virtù'. Nella continuazione del commento di Tommaso ripete la definizione Pietro D'Alvernia, In Politic. cont. IV lect. 7, 3: «nobilitas est virtus, idest inclinatio ad virtutem, et divitiae antiquae», e la ripete infine anche Dante, Mon. II III 4: «Est enim nobilitas virtus et divitie antique, iuxta Philosophum in Politicis». È certo anche che nella corte di Federico II della nobiltà si discutesse, anche se in termini diversi da quelli danteschi, come mostra l'interessante contentio di un autore rimasto anonimo (forse Terrisio di Atina) che invia il testo e chiede ulteriori lumi ai due giudici imperiali Pier della Vigna e Taddeo di Sessa: vd. Fulvio Delle Donne, «Una disputa sulla nobiltà alla corte di Federico II di Svevia», Medioevo romanzo, XXIII, 1999, p. 3-20 (il testo, p. 16-20). 
quanto essa è «regolatrice e rettrice di tutte le nostre operazioni, giustamente» (ibid. Ix 1). E lasciamo anche, per brevità, che il libro quarto del Convivio da sùbito trasformi l'improvviso e intenso squillo ghibellino del De vulgari eloquentia in una argomentazione filo-imperiale appoggiata al mito della romanità, come Scott ha ben segnalato, già saldamente fondata sui nodi concettuali e politici che sarà poi il Monarchia a riprendere e sviluppare. Lasciamo tutto questo, ripeto, che in ogni caso conferma come in quegli anni, 1304-1306, la svolta ghibellina fosse ormai pienamente attuata, e torniamo a Le dolci rime per osservare come la polemica contro il parere dell'imperatore (come chiarirà bene il Convivio, quel «secondo il suo parere» è pregnante) non metta per nulla in discussione l'istituto imperiale in quanto tale, e semmai dia per scontato che Federico II godesse tanto dell' "antica possession d'avere» quanto dei «reggimenti belli», dal momento che sono stati poi altri meno sapienti di lui che dalla definizione hanno eliminato $\mathrm{i}$ «reggimenti belli» che probabilmente non avevano, e l'hanno ridotta al solo possesso delle ricchezze. La canzone, insomma, non ha in sé nulla di anti-imperiale, come pure talvolta s'è pensato: di più, l'opinione dell'imperatore è pur sempre la più autorevole di tutte, e quella, dunque, con la quale occorre confrontarsi. Altrettanto certamente non ha nulla di filo-guelfo, ed anzi è di particolare interesse per il nostro discorso che invece corrisponda «sul piano dell'elaborazione dottrinaria e dell'elaborazione dottrinale, all'esigenza politica e sociale di mitigare il rigore degli Ordinamenti di giustizia, i quali, portando a compimento quell'autentica rivoluzione che fu l'instaurazione del Comune di Popolo, avevano trasformato il segno della prelatura - la nobilitas appunto- in motivo di esclusione dalla vita civile», e che «rileggendo e definendo in prospettiva etica i concetti di gentilzza e di leggiadria, il poeta invita i populares a riconsiderare le proprie posizioni più radicali, promuovendo allo stesso tempo nei magnates una riforma dei loro mores più asociali e pericolosi». ${ }^{40}$

La leggiadria, appunto: è infatti in suo nome che la polemica anti-popolare da implicita si fa esplicita, e assai dura, nel caso della successiva Poscia ch'Amor, che per la data di composizione va ragionevolmente accoppiata a $L e$ dolci rime. Qui Dante dà una propria definizione della leggiadria, una qualità cortese che non è tout court una virtù, perché, come Dante spiega, la virtù assolutamente considerata s'addice a tutti, religiosi e filosofi compresi, mentre la leggiadria, così come egli l'intende, è una virtù mista integralmente laica che deve informare il vasto arco dei comportamenti sociali, anche quelli amorosi, di quella che oggi diremmo la classe dirigente, o in altri termini, e con varie semplificazioni, la ricca borghesia che governava il comune fiorentino. ${ }^{41}$ Dante quindi raccomanda ai ceti emergenti del comune un modo di essere elegante

40. Così Borsa, «Sub nomine nobilitatis», cit., p. 66 e 77.

41. Per quanto qui riassumo, rimando all'ampia lettura nella quale ancora mi riconosco: Enrico FenzI, "Sollazzo" e "Leggiadria”. Un”interpretazione della canzone dantesca "Poscia ch'Amor"”, Studi danteschi, LXIII, 1991 [ma 1997], p. 191-280, e alle ricche note di commento di Claudio Giunta, in Dante, Opere, Milano: Mondadori, 2011, I, p. 326-354. 
e coltivato che assuma il meglio del modello aristocratico, passato al filtro di un'etica tutta civile e urbana: in altre parole, prefigura un ideale di eccellenza umana che passerà intatto dai comportamenti sociali a quelli specificamente linguistici raccomandati dieci anni dopo nel De vulgari eloquentia. Anche in questo caso, molto sarebbe da dire. Per esempio, è significativo che Dante agisca sul lessico con espliciti intenti di novità, connotando in senso positivo una leggiadria che nella tradizione anche a lui vicina non aveva solo il significato d'origine provenzale di 'leggerezza', 'capriccio', ma marcava anche l'arroganza dei nobili, sì che la nuova attribuzione di senso al termine ben corrisponde al tentativo di trasferire i valori migliori dell' ethos nobiliare a un nuovo e composito strato sociale che deve finalmente imparare a comportarsi in maniera adeguata al suo potere e alla sua ricchezza. Restando tuttavia al filo del nostro discorso, spicca un particolare la cui portata forse non è stata sin qui ben meditata. Afferma Dante, v. 11-14, che la leggiadria è «nome di valore», ed è
bella tanto
che fa degno di manto
imperial colui dov'ella regna:
ell'è verace insegna
la qual dimostra u' la vertù dimora.

La dignità e la virtù imperiale sono dunque additate come la pietra di paragone, il metro assoluto che riesce a mostrare quale sia l'altissimo valore della leggiadria. Per la metafora, si può allegare il Tesoretto, 34-35:

che voi corona e manto

portate di franchezza,

ove l'espressione di lode suona particolarmente adatta se, come penso, il dedicatario dell'opera è Luigi IX, re di Francia. ${ }^{42}$ L'espressione di Brunetto rientra nel tipo abbastanza frequente del portare $\mathrm{o}$ avere manto (di umiltà, di gioia, ecc.) secondo il traslato biblico dell'indossare e quindi del possedere. ${ }^{43} \mathrm{Ma}$ in Dante c'è qualcosa di più intenso, perché l'aggettivo fa aggio sul sostantivo, e l'uomo leggiadro diventa degno d'essere fatto imperatore. A conferma sta il fatto che il manto imperiale non è solo una metafora, perché il Weltenmantel aureo, preziosamente ricamato con figurazioni astrali, era un simbolo concreto,

42. Vd. per ciò FenzI, «Brunetto Latini», cit., p. 330-331 e nota 12.

43. Job 29, 14: «Iustitia indutus sum»; Paolo, Coloss. 3, 12, ecc. Per altre occorrenze della metafora vd. Gianfranco Contini, in Poeti del Duecento, Milano-Napoli: Ricciardi, II, p. 586, per Lapo Gianni, VIII, Amore i’ prego 13 e 24, e Rosanna BetTARIni per Dante da Maiano, XXXIII, Già non porà 3-4, nell'edizione delle sue rime, Firenze: Le Monnier, 1969, p. 97-98. In particolare, vd. Chiaro Davanzati, XXVII, Chiunque altrui blasma 91-92: «Imperial coron'ha veramente / di tutta la bieltate», e soprattutto Dante, Epist. I 7: «tante urbis misericordiam induistis»; ibid. II 6: «mores eius egregios induatis» (vi siete rivestito di misericordia per così grande città; vi rivestiate dei suoi egregi costumi). 
come la corona, lo scettro, il trono, della somma autorità imperiale. ${ }^{44}$ Il che suggerisce almeno due considerazioni: la prima, che questo rapido cenno stia in sottile rapporto con la citazione dell'opinione imperiale circa la definizione della nobiltà nella seconda stanza di Le dolci rime, forse come correttivo interno di quella polemica e insieme come larga definizione dell'orizzonte massimo, laico e civile, nel quale il dibattito dantesco si iscrive; la seconda, che tale iperbolico elogio definisca un ideale umano di vasta e compiuta perfezione riassunto dall'intima corrispondenza tra manto e insegna: come il manto è insegna/segno dell'autorità imperiale, così la leggiadria è insegna/segno della virtù in atto. E le due insegne convengono in sommo grado l'una all'altra, come la virtù conviene all'imperatore e viceversa.

Ma di là da una siffatta immagine imperiale che in sé compendia quell'ideale, all'altro capo cosa sta? La risposta deve essere netta: niente e nessuno. Questa infatti è la realtà che Dante stesso denuncia nella canzone, là dove confessa di non sapere per chi mai egli stia parlando: «tratterò il ver di lei [la leggiadria], ma non so cui» (v. 69), e alla fine, nell'ultimo lapidario verso della settima stanza: «Color che vivon fanno tutti contra». Così, egli non solo spiega perché, mancando i destinatari, necessariamente debba mancare anche il congedo, ma soprattutto prende atto di una situazione che distrugge in radice le ragioni stesse della canzone e, a ritroso, la cancella. Parla, ma non ha ascoltatori; insegna, ma non ha discepoli ... Se da una parte sta la sublime, aristocratica immagine della virtù imperiale, dall'altra non sta affatto, a bilanciarla con valori suoi propri, una sua possibile riedizione borghese: piuttosto, se tutti i viventi 'fanno contra', ci saranno i fiorentini di sempre: arroganti, esibizionisti, golosi, lussuriosi, sciocchi, quali quelli vituperati nella prima parte della canzone, che non sanno cosa sia l'amore e «come al furto il ladro, / così vanno a pigliar villan diletto", con donne "che paiono animal sanza intelletto» (v. 53-57).

La canzone conserva in più di un luogo l'eco dell'atteggiamento didattico brunettiano, ma sicuramente e consapevolmente distrugge la base stessa, guelfa e repubblicana, alla quale si riferiva l'ideologia di Brunetto, rispetto al quale quelle affermazioni negative suonerebbero contraddittorie e inconcepibili. Il punto non è da poco perché il fatto che il Dante 'comunale' del 1295 non riesca a ravvisare nei concittadini la naturale controparte con la quale condividere un discorso etico-comportamentale importa di per sé una fuoruscita dal solco rigorosamente guelfo del maestro, e l'affacciarsi su una situazione di personale isolamento. Per quanto in maniera lieve, direi innegabile che a leggere quel: "tratterò il ver di lei [la leggiadria], ma non so cui", e il verso che chiude la canzone: "Color che vivon fanno tutti contra» si avverta già il senso generale di una solitudine assai prossima a quella che più o meno dieci anni

44. Per tale mantello si vedano i lavori di Rudolf Eisler e Percyr Ernst Schramm citati in Enrico FenzI, "Di alcuni palazzi, cupole e planetari nella letteratura classica e medievale e nell'“Africa" del Petrarca» (1976), ora in ID., Saggi petrarcheschi, Firenze: Cadmo, 2003, p. 229-303: p. 290 nota 96 e 300-301. Ma vd. pure Fritz SAXL, Le fede negli astri. Dall'antichità al Rinascimento, a cura di Salvatore SetTis, Torino: Boringhieri, $\left(1985^{1}\right)$ 2007, p. 177. 
dopo lo deciderà a fare parte per sé stesso. Ma più precisamente ancora, e ancora qualche anno dopo è l'incontro infernale con Brunetto che suona come definitiva conferma della valenza anti-comunale di quei versi di Poscia ch'Amor, e conferisce loro il sapore della profezia. La condanna del vecchio maestro, nel quindicesimo dell'Inferno, indubbiamente basata sulla sua sodomia, contiene molte cose: tra esse, la condanna senza appello di quel Comune e di quel fallimentare progetto di educazione politica condotto in chiave strettamente municipale. Introducendo la parte sulla retorica nel Tresor, I 1, 8, ricamando ancora sull'eloquenza quale fondatrice della civiltà, scrive Brunetto che Anfione con le sue bonnes paroles «retraist les homes des sauvages roches ou il abitoient, et les amena a la comune habitation de cele cité». Ma per Dante la retorica delle buone parole non ha affatto funzionato, almeno a Firenze, e Brunetto gli avrà sì insegnato tante cose, ma il suo magistero ha lasciato tutto come stava, visto che proprio a lui, in una sorta di amara e rancorosa retractatio, è fatto dire: "quello ingrato popolo maligno / che discese di Fiesole ab antico, / [...] tiene ancor del monte e del macigno" (Inf. XV 61-63: corsivi miei). Di là dalla persona è dunque condannato un progetto, ed è condannato il ceto che avrebbe dovuto farsene carico: quel ceto di mercanti e banchieri e faccendieri ch'era la naturale base sociale e politica di Brunetto alla quale Roberta Cella ha dato nomi e cognomi ${ }^{45}$ e che ritroviamo in buona parte tra i sodomiti, gli scialaquatori e i suicidi dell' Inferno, lasciati ai loro vizi privati e (quando ci sono) alle loro pubbliche virtù. Il distacco di Dante è lucido e definitivo: quel mondo e quell'esperienza hanno dato quel che potevano, tanto o poco che sia, e nulla della sua effettuale e storica consistenza può ormai essere salvato. A queste ultime considerazioni, già argomentate altrove, ${ }^{46}$ s'aggiunga ora un importante supplemento d'analisi di Claudia Villa, dal quale giova citare ampiamente:

In questa città infernale, che ripropone fedelmente coscienza di classe e distinzioni fra gruppi di potere, il cancelliere Brunetto è ancora, come in vita, un protagonista, auctor di un progetto pur sempre affidato al suo Tresor: e se l'intenzione di Dante è quella di valutare, attraverso l'esperienza del maestro, la società in cui il vecchio priore ha operato, sembra di poter suggerire che si ridefinisca qui un discorso più complesso su tutte le forze che agirono all'interno dell'esperienza comunale. La città di Brunetto, collocata in una landa deserta e infuocata, risolta in un sabbione ardente privo di costruzioni, s'impone come una straordinaria metafora della forma di vita civile, garantita da istituzioni cittadine, rappresentata dal comune in cui il dettatore ancora credeva [...]. Se la riflessione politica di Dante, nella composizione dell'Inferno, sembra progressivamente avviata verso la negazione di ogni possibilità di vita civile all'interno della forma-comune ...

45. Roberta Cella, «Gli atti rogati da Brunetto Latini in Francia (tra politica e mercatura con qualche implicazione letteraria)", Nuova rivista di letteratura italiana, VI, 2003, p. 367-408. Se ne veda la recensione di Irene Maffia SCARIati in Studi e problemi di critica testuale, n. 71, 2005, p. 245-251, importante perché la studiosa ne ricava suggestive linee di ricerca relative alla condanna dantesca.

46. FenZI, «Brunetto Latini», cit., in part. p. 368-369. 
Nel suo viaggio — continua la Villa — Dante procede a saldare i conti con le scelte politiche e civili delle quali ha fatto esperienza, e verifica come fra arenghe e istruzioni ai podestà, il progetto politico di Brunetto si sia tragicamente arenato. E Brunetto per primo

deve riconoscere nel figlio la propria disfatta: poiché Dante è ormai un condannato a morte, un intellettuale che non tornerà mai più a Firenze e il populus, allevatosi alle passioni, non è che un gregge di bestie. ${ }^{47}$

Un gregge di bestie, aggiungiamo, immediatamente contrapposto (Inf. XV 73-77) alla «sementa santa» dei romani detentori dell'Impero, il "populus ille sanctus pius et gloriosus» di Mon. II v 5. Se le cose stanno così, ebbene, l'immagine corrente di Dante che avrebbe dimenticato o nascosto nell' Inferno la sua incipiente conversione ghibellina va assai sfumata e corretta, visto che una siffatta definitiva condanna della Firenze comunale e del suo ideologo è in re una aperta professione di anti-guelfismo. E una professione, per di più, che non giunge inattesa, perché quelle dichiarazioni di Poscia ch'Amor valgono come una precoce e impietosa diagnosi, e già mostrano un disagio profondo verso la società comunale.

5. Per concludere, dopo aver visto una sorta di Dante cripto-ghibellino già nelle canzoni 'dottrinali' del 1295, restiamo brevemente all'Inferno. In esso, è stato detto, la linea ghibellina emersa negli anni 1302-1304 si sarebbe interrotta, per riapparire finalmente robusta ed egemone nel Purgatorio. È davvero così? Personalmente, credo che le cose siano un poco più complesse, e bastano poche osservazioni a mostrarlo. Cominciamo con un caso che proprio Carpi solleva. In Inf. II 16-24 è detto che Iddio avrebbe concesso ad Enea di scendere da vivo agli Inferi, come Virgilio narra nel sesto dell'Eneide, in considerazione del grande scopo al quale il suo viaggio era ordinato, la fondazione di Roma e del suo impero:

Però, se l'avversario d'ogne mal cortese i fu, pensando l'alto effetto ch'uscir dovea di lui, e 'l chi e 'l quale, non pare indegno ad omo d'intelletto; ch'e' fu de l'alma Roma e di suo impero ne l'empireo del ciel per padre eletto: la quale e 'l quale, a voler dir lo vero, fu stabilita per lo loco santo ù' siede il successor del maggior Piero.

Quella sorta di precisazione finale, «a voler dir lo vero», fa un passo avanti rispetto all'Eneide e per dir così la completa alla luce di quella Verità rivela-

47. Claudia Villa, «Natura e corpo sociale. Retorica (e cecità) di ser Brunetto», Rivista di studi danteschi, X, 2010, p. 233-249: p. 238-239 e 346-347. 
ta che a Virgilio rimase inaccessibile: ${ }^{48}$ ma quello che segue s'è prestato a interpretazioni divergenti nel merito delle quali sarebbe lungo entrare. Possono tuttavia essere oggi ben esemplificate attraverso il modo di intendere di Giorgio Inglese il quale non vi vede alcuna impegnativa affermazione della superiorità papale quale culmine del processo storico voluto dalla Provvidenza per Roma, ma piuttosto il contrario. Egli intende infatti che l'Impero fu stabilito quale giovamento e sostegno alla missione della Chiesa, senza revocare in dubbio l'idea-guida di Dante che all'autonoma azione dell'Impero spettasse di assicurare al genere umano la felicità naturale su questa terra. ${ }^{49} \mathrm{Si}$ può anche aggiungere che vicino a questi versi dell' Inferno anche per varie corrispondenze lessicali è il Convivio: si veda in particolare IV v 3-6, né ciò contrasta con la dominante 'imperiale' del libro. ${ }^{50} \mathrm{E}$ ancora nella primavera del 1314 , e cioè in un'epoca di ormai conclamata fede ghibellina, Dante non diceva nulla di diverso nell' Epistola ai cardinali:

Romam — cui, post tot triumphorum pompas, et verbo et opere Christus orbis confirmavit imperium, quam etiam ille Petrus, et Paulus gentium predicator, in apostolicam sedem aspergine proprii sanguinis consecravit, ${ }^{51}$

e che è ben Pietro che in Par. XXVII 23-24, grida il proprio sdegno per l'usurpazione' da parte di Bonifacio VIII del soglio pontificio: "Quelli ch'usurpa in terra il luogo mio, / il luogo mio, il luogo mio ...", ove il luogo mio è

48. Siro A. Chimenz, "Canto II», in Letture dantesche. Inferno, a cura di Giovanni Getto, Firenze, Sansoni, 1955, p. 29: «L'inciso è un avvertimento — discreto ma evidente- a Virgilio, pagano e cantore di Roma pagana». E allo stesso proposito Michelangelo PiCONE, "Inferno II: 1'“altro viaggio"”, in Sotto il segno di Dante. Scritti in onore di Francesco Mazzoni, a cura di Leonella Coglievina e Domenico De Robertis, Firenze: Le Lettere, 1998, p. 249-260: p. 257: «Nel momento stesso in cui cita Virgilio, Dante lo corregge e lo integra: l'Eneide trova nella Commedia il suo completamento e inveramento finale».

49. D. A., Commedia, revisione del testo e commento di Giorgio InGLESE. Inferno, Roma: Carocci, 2007, p. 53. Per parte sua Torraca, ad loc., insinuava a proposito di «a voler dir lo vero": "sembrerebbe una concessione fatta un po' a malincuore».

50. Conv. IV v 3-6: «eletto fu in quello altissimo e congiuntissimo consistorio della Trinitade che 'l Figliuolo di Dio in terra discendesse a fare questa concordia. E però che nella sua venuta lo mondo, non solamente lo cielo ma la terra, convenia essere in ottima disposizione; e la ottima disposizione della terra sia quando ella è monarchia, cioè tutta ad uno principe [...] ordinato fu per lo divino provedimento quello popolo e quella cittade che ciò dovea compiere, cioè la gloriosa Roma. E però che anche l'albergo dove 'l celestiale rege intrare dovea, convenia essere mondissimo e purissimo, ordinata fu una progenie santissima [...] e questa progenie fu quella di David, del qual discese la baldezza e l'onore dell'umana generazione, cioè Maria [...] E tutto questo fu in uno temporale, che David nacque e nacque Roma, cioè che Enea venne di Troia in Italia, che fu origine della cittade romana, sì come testimoniano le scritture. Per che assai è manifesto la divina elezione del romano imperio, per lo nascimento della santa cittade, che fu contemporaneo alla radice della progenie di Maria».

51. Epist. XI 3 [Roma - alla quale, dopo la pompa di tanti trionfi, Cristo con le parole e con i fatti confermò l'impero del mondo, e che anche Pietro e Paolo, il predicatore delle genti, consacrarono quale sede apostolica con l'aspersione del proprio sangue]. 
sì, prima di tutto, la funzione di pontefice, ma pure il luogo della tomba di Pietro e infine la stessa Roma, il loco santo, appunto, «ù siede 'l successor del maggior Piero".

Diversa invece è ora l'interpretazione di Carpi, per il quale quei versi dell' Inferno appaiono come clamorosa, indiscutibile affermazione di fede guel$\mathrm{fa}$, a un punto tale da condurre a due conclusioni obbligate. La prima: Dante avrebbe abbandonato le convinzioni filo-imperiali acquisite e argomentate nel De vulgari eloquentia e nel Convivio, costringendoci a immaginare, scrive Carpi, «un percorso ideologico a dir poco accidentato e tortuoso; oppure, che è la mia ferma convinzione, il luogo effettivamente guelfo di Inf. II appartiene a quella precedente stesura dei primi canti in Firenze, poi recapitatigli da Dino Frescobaldi in Lunigiana, come narra il Boccaccio» (p. 4).

Mi sembra che le due ipotesi prospettate siano entrambe eccessive, ed eccessivo sia in particolare il loro comune punto di partenza. Non vedo come sia specialmente guelfa l'affermazione, in Dante costantemente ripetuta, che lo stesso decreto divino che ha portato a Roma imperiale avesse anche sancito il suo ruolo di capitale della cristianità, e non vedo nulla di specialmente impegnativo nella correlata e ovvia dichiarazione che essa sia la sede papale. Il presente siede, che s'oppone ai vari fu che precedono, sancisce, direi, la neutralità della circostanza. Tra l'altro, se ci atteniamo al tempo fittizio del racconto, come Dante in genere fa, chi siede è Bonifacio VIII; se al tempo reale della scrittura, è Clemente V, il Guasco, cioè il papa che Dante disprezzò in maniera profonda e costante. Nell'un caso e nell'altro sembra insomma difficile che le parole di Dante comportino ch'egli si dichiari «ortodosso seguace della pars Ecclesiae», come ancora Carpi scrive. Ma non voglio insistere, anche per non ripetere il vecchio ma sempre pertinente dibattito tra Parodi, Nardi e Francesco Ercole. Parodi sosteneva che nell'Inferno si stende un alto silenzio sull'Impero e che la visione provvidenziale che ne fa una sorta di precondizione della rivelazione cristiana tiene campo sino al momento dell'impresa di Arrigo VII, che segna il vero punto di svolta oltre il quale si può parlare di un Dante ghibellino. Nardi, facendo perno insieme su Virgilio e sul Veltro e complicando le cose con la tesi che voleva la Monarchia composta dopo il Convivio e prima della Commedia, addirittura rovescia questo schema proponendo una scansione dell'esperienza dantesca che debbo riferire per far capire quale sia, oltre tanti particolari, la posta in gioco. Conclude dunque Nardi:

La Monarchia, dunque, è posteriore al Convivio, ma anche anteriore all' Inferno. Nell'opera politica la logica che trasporta lo scrittore a definire la base filosofica dell'indipendenza dell'Impero dalla Chiesa, lo conduce a stabilire, implicitamente, un ugual rapporto d'indipendenza della ragione dalla fede, della perfezione naturale dell'umanità da quella soprannaturale. Il Virgilio della Monarchia [...] non attende nessuna Beatrice. Il rapporto medievale di dipendenza della ragione dalla fede, della "philosophia ancilla theologiae», è negato non meno di quello, asserito dai decretalisti, di soggezione dell'Impero al Papato. Ma questa affermazione dell'indipendenza della ragione umana manca, nel pensiero dantesco, di ogni ulteriore sviluppo, ed è, anzi, subito attenuata e svalutata colle parole $[. .$. 
con cui finisce il trattato. Nelle quali parole è preannunziata la Commedia; ché nel Poema, pur mantenendosi la sostanza del pensiero politico quanto alla missione dell'Imperatore, l'ordine dei rapporti tra la ragione e la fede vien ristabilito secondo il concetto teologico tradizionale, e Virgilio, simbolo della ragione umana, diventa messo e araldo di Beatrice, simbolo del pensiero divino. È probabile che, quando Dante ebbe concepito il disegno generale della Commedia, sentisse il bisogno, per l'esecuzione di esso, di approfondire i problemi teologici che avrebbe incontrato per via. E da un più accurato studio della teologia medievale egli fu certamente indotto a ristabilire la subordinazione della ragione alla fede, della filosofia alla teologia. Ma in tal modo la tesi del terzo libro della Monarchia perdeva la sola possibile giustificazione razionale e rimaneva una semplice dissonanza nell'armonia del pensiero filosofico dello stesso Poeta, una screpolatura nella salda compagine dell'edifizio teologico del Medio Evo. L'assoluta autonomia dello Stato attendeva di esser difesa da chi avesse svolto, senza pentimenti, il concetto dei «philosophica documenta" della Monarchia. ${ }^{52}$

Questo schema sicuramente ha il pregio della semplicità e linearità, se lo riduciamo all'essenziale cammino dalla ragione alla fede nel corso del quale il laicismo filosofico e politico (quello che sarebbe stato raccolto e sviluppato da Machiavelli) viene finalmente ricompreso e superato dal 'pensiero divino' della Commedia. Ma tale schema non ha avuto fortuna, e in particolare, per quanto qui interessa, non è stata accolta l'ipotesi (ma per Nardi una certezza) che tutto il Dante che diremmo per comodità ghibellino, che sostiene l'indipendenza dell'Impero dalla Chiesa e l'indipendenza della ragione dalla fede, faccia blocco a sé, chiuso sulla terna De vulgari eloquentia, Convivio e Monarchia, alla quale succede la nuova trascendenza cristiana della Commedia.

Ho sopra accennato alla complessità della posta in gioco, che spicca se confrontiamo lo schema di Nardi con quello odierno e opposto al quale, abbiamo visto, Santagata in gran parte s'attiene e riveste di forma narrativa. Diverso dall'uno e dall'altro è lo schema di Carpi che pure tiene ferma la dominate guelfa dell' Inferno e proprio per questo s'adopera a capire e sanare

52. Bruno NARDI, «Il concetto dell'Impero nello svolgimento del pensiero dantesco» (1921), in ID., Saggi di filosofia dantesca, Firenze: La Nuova Italia, 1967, p. 215-275: p. 274-275. Ho citato le frasi conclusive del lungo saggio: le stesse conclusioni sono ripetute in fine al saggio che segue nello stesso volume: Tre pretese fasi del pensiero politico di Dante, p. 276310, nel quale Nardi contesta le tesi di Francesco ErCole, «Le tre fasi del pensiero politico di Dante» (1921), in ID., Il pensiero politico di Dante, Milano: Alpes, 1928, p. 271-407, che a sua volta contestava puntualmente le sue e precisamente scioglieva quel 'blocco' negando che vi fosse opposizione tra la Commedia e la Monarchia, che sarebbe stata composta dopo la morte di Arrigo VII, dunque dopo il 1313, in concomitanza con la fine del Purgatorio. Il saggio di Ernesto Giacomo Parodi discusso da Nardi è «Del concetto dell'Impero in Dante e del suo averroismo", Bullettino della Sociertà Dantesca Italiana, XXVI, 1919, p. 105-148. Per quanto riguarda la discussa data della Monarchia, vd. una rassegna delle varie proposte in Enrico FENZI, «È la "Monarchia" l'ultima opera di Dante? (a proposito di una recente edizione)", Studi danteschi, LXXII, 2007, p. 215-238 (l'edizione è quella a cura di Francesco Furlan, Milano: Mondadori [Biblioteca dell'Utopia], 2004). 
le contraddizioni che ne nascono: ma sarebbe meglio dire l'anti-schema, visto che il tentativo e propriamente la scommessa dello studioso è quella di rinunciare ai 'blocchi' di qualsiasi tipo, e di ricostruire sin dove possibile un percorso accidentato quanto si voglia ma alla fin fine unitario, che cresce su di sé e nel quale, volta per volta, tout se tient. Proprio in questa logica di fondo che mi vede profondamente solidale ripeto dunque che non intendo andar oltre nel discutere di quei versi del secondo dell'Inferno. Sia come sia, infatti, dobbiamo impedire che la paglia ci impedisca di vedere la trave: in una parola, il fatto che l'Inferno per quanto attiene alla visione politica si apra con Virgilio, e nel caso con il Virgilio che a suo tempo ha celebrato l'eternità dell'Impero, ${ }^{53}$ e ora immediatamente si fa profeta del Veltro, e torna a celebrare Enea protetto da Dio per "l'alto effetto" che dal suo viaggio era destinato che sortisse, dal momento "ch'e' fu de l'alma Roma e di suo impero / ne l'empireo del ciel per padre eletto" (e per tale 'elezione' basti qui il rinvio a Conv. IV Iv 8-14). L'Inferno, comincia, insomma, all'insegna di una visione perfettamente e autonomamente imperiale, di lì a poco confermata dal trovare tra gli spiriti magni del Limbo «Cesare armato con li occhi grifagni» (Inf. IV 123), salvato dunque quale fondatore dell'Impero, e collocato nel nobile castello «difeso intorno d'un bel fiumicello» e dotato all'interno di un "prato di fresca verdura». Sì che, lo si giudichi come si vuole, non sarà certo quello scrupolo inteso a «voler dir lo vero" a ribaltare quella fondante e generale dimensione e scelta politica che l'Inferno non solo non smentirà mai, ma finirà invece per confermare in maniera addirittura clamorosa. ${ }^{54}$

Si considerino infatti ancora due punti toccati da un Carpi che prima che con altri discute con se stesso. Dapprima egli, preoccupandosi, come s'è appena sopra accennato, di tenere insieme tutti i fili di un percorso fortemente articolato ma unitario, osserva che Dante nel corso dell'Inferno aveva ben in mente il discorso politico avviato nei due trattati, «del che è visibile spia il Federico II di Pier delle Vigne (Inf. XIII 55-78)»: e poco sopra giustamente scrive:

nelle parole di Pier delle Vigne, un tono di sacrale venerazione e per Federico e per la corte: l'onore di cui Federico è riconosciuto degno e il glorioso che spetta al suo servizio pertengono alla medesima area concettuale e semantica

53. All'interno di una breve ma intensa 'lettura' nella quale torna a ribadire le proprie posizioni, Nardi ancora sottolinea come il Virgilio dantesco sia non solo poeta «ma anche filosofo e storico verace, più dello stesso Livio, a narrare le origini divine dell'Impero romano e la predestinazione di esso alla signoria del mondo» ("Tre momenti dell’incontro di Dante con Virgilio» [1965], in ID., Saggi e note di critica dantesca, Milano-Napoli: Ricciardi, 1966, p. 220-237: p. 230.

54. Una volta accertato, come lo stesso Carpi fa notare, che nell'Inferno non c'è mezza parola contro l'Impero, a conferma può essere significativo che là dove è questione dei tiranni che "dier nel sangue e nell'aver di piglio" manchi appunto ogni figura imperiale, quale per esempio Nerone, e che tale categoria di dannati sia rappresentata nell'ordine da Alessandro di Fere, Dionigi di Siracusa, Ezzelino da Romano, Obizzo II d'Este. 
della nobiltatem, della rectitudinem, degli humana attribuitigli in De vulgari I 12. Glorioso, fra parentesi, è lemma frequente nel paradiso, invece quasi apax nell'Inferno [...]. Insomma, nel lessico di Dante il glorioso offizio suona molto forte, l'Impero esige parole di paradiso. ${ }^{55}$

Poi, mette in forte evidenza la chiusura infernale su Bruto e Cassio traditori di Cesare, maciullati con Giuda nelle fauci di Lucifero, a dire che «i traditori di Cesare valgono i traditori di Cristo» (p. 17-18). ${ }^{56}$ Ma non si tratta di un'altra eccezione, come mi pare che alla fin fine intenda Carpi, che colloca questi casi in controtendenza. È invece una iniziativa, questa di Dante, che salda il cerchio aperto con i primi canti, serrando l'intera cantica tra due estremi che si richiamano a distanza e mostrano la continuità, non la frattura o la sospensione, della riflessione dantesca, e la sua capacità strutturante. Con ciò resta vero che si possa parlare in generale di un Inferno guelfo, ma essenzialmente perché la guelfa Firenze è la città infernale per eccellenza, ${ }^{57}$ come Dante ripetutamente denuncia per tutta la cantica: città di Marte travagliata da odi e guerre senza fine, preda costante di un incredibile caos civile e politico ch'è l'esatto, effettuale opposto di quello che avrebbe voluto l'ideologia comunale di Brunetto. Sì che infine, se torniamo a ripensare al senso politico dell'incontro che Dante ha con il vecchio maestro, non sarà solo una battuta il dire che sì, l'Inferno è davvero guelfo perché Dante in esso rappresenta un mondo privo dell'Impero, e mette in scena la tragedia della sua assenza.

55. Carpi, «Federico II e Manfredi», cit., p. 13-15.

56. La parte finale del discorso di Carpi è dedicata alla questione del diverso trattamento che Dante riserva a Bruto e Cassio, regicidi equiparati a Giuda, e a Catone «eroe purgatoriale della libertà civile», in implicito dialogo — mi sembra — con l'importantissimo saggio di Giuliano Tanturli, "Continuità dell'umanesimo civile da Brunetto Latini a Leonardo Bruni», in Gli umanesimi medievali. Atti [...], a cura di Claudio Leonardi, SISMELEdizioni del Galluzzo, 1998, p. 736-780, il quale discute della insanabile contraddizione dantesca «fra l'ossequio a Cesare e all'impero e l'apprezzamento dei valori e degli eroi repubblicani», e colloca tale contraddizione nella lunga prospettiva che da Brunetto a Leonardo Bruni vede via via crescere la valutazione negativa dell'impero e quella positiva del modello rebubblicano, che diventa l'ideologia ufficiale del cosiddetto 'umanesimo civile'.

57. Vd. al proposito il rapido ed efficace consuntivo di Amilcare A. IANnUCCI, «Firenze città infernale», in Dante. Da Firenze all'aldilà. Atti [...], a cura di Michelangelo Picone, Firenze: Cesati, 2001, p. 217-232. E si legga ora il bel volume di Elisa BriLli, Firenze e il profeta. Dante fra teologia e politica, Roma: Carocci, 2012, in particolare l'ampio capitolo 2, «La 'civitas diaboli sub specie Florentiae'», p. 121-270. 